\title{
1 Traceback of Core Transcription Factors for Soybean Root Growth Maintenance under
}

2 Water Deficit

Li Lin ${ }^{1}$, Jan Van de Velde ${ }^{2}$, Na Nguyen ${ }^{1}$, Rick Meyer ${ }^{3}$, Yong-qiang Charles $\mathrm{An}^{3}$, Li Song ${ }^{1}$, Babu Valliyodan $^{1}$, Silvas Prince ${ }^{1}$, Jinrong Wan ${ }^{1}$, Mackensie C Murphy ${ }^{1}$, Eiru Kim ${ }^{4}$, Insuk Lee ${ }^{4}$, Genevieve Pentecost ${ }^{1}$, Chengsong Zhu ${ }^{1}$, Garima Kushwaha ${ }^{5}$, Trupti Joshi ${ }^{5,6,7}$, Wei Chen ${ }^{1}$, Gunvant Patil ${ }^{1}$, Raymond Mutava ${ }^{1}$, Dong $\mathrm{Xu}^{5,6}$, Klaas Vandepoele ${ }^{2}$ and Henry T. Nguyen ${ }^{*}$

1. Division of Plant Sciences and National Center for Soybean Biotechnology, University of Missouri, Columbia, MO 65211, USA

2. VIB Center for Plant Systems Biology, Department of Plant Biotechnology and Bioinformatics, Technology park 927, Ghent University, B-9052 Ghent, Belgium

3. USDA-ARS, Plant Genetics Research Unit at Donald Danforth Plant Sciences Center, 975 N Warson Road, St. Louis, MO 63132, USA

4. Department of Biotechnology, College of Life Science and Biotechnology, Yonsei University, Seoul 120-749, Korea

5. Informatics Institute and Christopher S. Bond Life Sciences Center, University of Missouri, Columbia, MO 65211, USA

6. Department of Computer Science, University of Missouri, Columbia, MO 65211, USA

7. Department of Molecular Microbiology and Immunology and Medical Research Office, School of Medicine, University of Missouri, Columbia, MO 65212, USA

Corresponding author: Henry T. Nguyen, nguyenhenry@missouri.edu

First author: Li Lin, lilin.dhs@gmail.com

\section{ABSTRACT}

Some crops inhibit shoot growth but maintain root growth under water-deficit conditions. Unraveling the molecular mechanisms of root plasticity under water deficit conditions in plants remains a major challenge. We developed an efficient platform for identifying core transcription factors (TFs) that collectively regulate each other and/or themselves in response to water stress, and exploring their interconnected regulatory circuitry involved in root growth maintenance under water deficit in soybean. We performed multi-species phylogenetic footprinting combined with spatial-temporal transcriptome analysis of soybean (Glycine max) roots under water deficit to identify conserved motifs that function in the water-stress response. Using these functional conserved cis-motifs, we applied a new approach to trace back motifs-associated core TFs ingroup 
40 as signal mediators, which mediate signaling between abiotic and endogenous stimuli. We

41 integrated a co-functional TF-TF network and conserved motif-centered TF-DNA networks to

42 construct a core TF network defined by mutual cross-regulation among core TFs. We found that

43 core TF ARG (Abscisic acid response element binding factor-like Root Growth regulator)

44 represses BRG (Brassinosteroid enhanced expression-like Root Growth regulator) expression

45 through binding to its promoter at a conserved binding site. ARG and BRG antagonistically

46 regulate Phytochrome-interacting factor-like Root Growth regulator (PRG) and combinatorially

47 regulate some other core TFs. These core TFs form complex regulatory circuits to integrate light

48 and multiple hormone signaling pathways and maintain root growth in response to varying degrees

49 of water stress. Our study provides valuable information to unravel the complicated mechanisms

50 of molecular networks involved in the regulation of root growth under water deficit.

51

52 Keywords: root, core TF, water deficit, soybean 


\section{BACKGROUND}

55 Drought is the primary cause of yield loss in agriculture worldwide. Understanding the development and architecture of roots, as well their plasticity, would pave the way for improving drought tolerance in crops and stabilizing productivity under drought conditions (1). Several agronomic species, such as soybean (Glycine max) and maize (Zea mays), exhibit an important adaptation to drought stress: substantial primary root elongation is maintained at low water potentials, whereas shoot growth completely stops (2). Unraveling the molecular mechanisms of root plasticity under water deficit conditions in plants remains a major challenge.

It has been demonstrated that $\mathrm{ABA}$ regulates root growth under osmotic stress conditions via an interacting hormonal network with cytokinin, ethylene and auxin (3). Since plant stress responses must be coordinated with growth and development, there is crosstalk between stress signaling pathways and both hormonal and growth/developmental signaling pathways (4). The integration of plant hormone signaling predominantly occurs at the transcriptional level (5). Transcription factors (TFs), which serve as major regulators of gene expression, play crucial roles in stress responses (6-8). TFs are often sites of signal convergence; signal-regulated TFs act in concert with other context-specific TFs and transcriptional co-regulators to establish sensory transcription regulatory networks in response to abiotic stress $(5,7,9)$.

Evidence suggests that multiple drought-responsive TFs interact. For example, AREB1, AREB2 and ABF3 bind to the promoter of DREB2A and induce its expression in an ABREdependent manner (10). In addition, DREB1A, DREB2A and DREB2C physically interact with AREB/ABF proteins (11), and crosstalk occurs between ABA-dependent and ABA-independent response pathways $(7,12)$. Furthermore, ABRE sequences are present in the promoter regions of stress-responsive NAC genes in Arabidopsis thaliana and rice (13), and NAC016 directly binds to the $A R E B 1$ promoter and represses its expression through a trifurcate feed-forward regulatory loop involving NAP during the drought stress response (14). DREB/CBF binds to a DRE/CRT motif in the ERF1 promoter under abiotic stress, and also to the GCC box in the promoter of ERF1 in response to biotic stress, suggesting that ERF1 might integrate ethylene, jasmonate and ABA

81 signaling and play an important role in biotic and abiotic stress responses (15). It has been

82 suggested that a highly interconnected TF gene regulatory network coordinates cell differentiation

83 in the Arabidopsis root (16). A recently constructed TF network provides a roadmap of ABA84 elicited transcriptional regulation by 21 ABA-related TFs in Arabidopsis seedlings (17). Despite 
the central roles of some TFs in water stress responses, the exact regulatory mechanisms of individual TFs and their interactions remain obscure.

Soybean has experienced a relatively recent whole-genome duplication (13 million years ago) (18). Compared to Arabidopsis, soybean has a higher total number of TFs and a greater proportion of some TF families, including some legume-specific subfamilies (18). TFs are often regulated by other TFs and/or by themselves, resulting in interconnected regulatory circuits within a large, complex regulatory network (19). Individual subcircuits within a Gene Regulatory Network (GRN) do not operate in a linear hierarchy but are instead strongly intertwined $(20,21)$. Some important regulatory information in GRNs is ultimately encoded in individual cis-regulatory elements (CREs) that modulate gene activities through their interaction with TFs (20). However, even in well-studied organisms, TF binding site information is available for relatively few TFs, and there are limited DNA binding data for TFs in the majority of eukaryotes (22).

Here, we aimed to identify a group of master TFs that mediate signaling between developmental programs and water stress. These core TFs collectively regulate each other and/or themselves, forming interconnected regulatory circuits to integrate multiple signaling pathway under water deficits in soybean primary root. We developed an efficient platform for identifying core TFs and exploring core regulatory circuitry involved in root growth maintenance under water deficit in soybean using the assembly strategy (Fig. $1 \mathrm{a}$ and b). We examined the spatial-temporal expression of soybean genes under water deficit and identified conserved functional CREs using multi-species phylogenetic footprinting. Since current cis-motif datasets are generalized for TF families and cannot associate individual TFs with specific cognate motifs, we thus applied a new approach to trace back core TFs ingroup associated with identified CREs. The regulatory subnetworks defined by mutual cross-regulation among groups of core TFs were further investigated through integration of information about co - functional links of core TFs, TF - DNA interaction, different gene function annotations and gene expression profiles. Finally, based on this network, we prioritized and functionally characterized several key regulators involved in the

\section{RESULTS}

\section{Transcript profiling of various regions of soybean roots in response to water stress}


115 Soybean roots continue to elongate when exposed to drought and exhibit region-specific responses

116 to low water potentials. We previously identified three regions of soybean primary roots based on

117 their growth under low water potential -1.6 MPa (23): region 1 (R1) exhibits the same elongation

118 rates under well-watered and water stress conditions; region 2 (R2) exhibits maximum elongation

119 rates in well-watered soil, but progressive deceleration in growth under water stress; and region 3

120 (R3) exhibits slow growth under well-watered conditions and no growth under water stress.

121 To explore the molecular mechanism underlying root elongation in response to water stress,

122 we set out to examine the expression of soybean genes in the three different regions (R1, R2 and

$123 \mathrm{R} 3$ ) of the root after $5 \mathrm{~h}$ and $48 \mathrm{~h}$ (root tip water potential had decreased to approximately -1.6

$124 \mathrm{MPa}$ by this time) of water stress treatment using an Affymetrix Soybean GeneChip containing

12537,500 G. max probe sets. Transcript expression was profiled in different regions of water-stressed

126 (WS) and well-watered (WW) roots at both $5 \mathrm{~h}$ and $48 \mathrm{~h}$ time points. Therefore, the transcripts were

127 divided into five groups according to their expression profiles across the comparisons (Fig. 2a,

128 Table S1): 5hR1WS vs 5hR1WW (D5hR1, D stands for differentiation); 48hR1WS vs 48hR1WW

129 (D48hR1); 5hR2WS vs 5hR2WW (D5hR2); 48hR2WS vs 48hR2WW (D48hR2); 48hR2WS vs

130 48hR3WW (D48hR2vR3). The last comparison between R2 of water-stressed roots and the zone

131 of growth deceleration in R3 well-watered roots distinguished stress-responsive genes in R2 from

132 those involved in cell maturation. Therefore, we reasoned that the 279 genes with more than two-

133 fold changes in expression detected in both D48hR2 and D48hR2vR3 were important (Fig. 2b;

134 Table S2), because these genes might indeed be regulated by water deficit conditions instead of

135 merely being associated with growth deceleration and tissue maturation closer to the apex.

136 Moreover, a comparison of this group of genes with significantly differentially expressed genes

137 identified in D5hR1, D48hR1 and D5hR2 revealed 49, 69 and 71 genes in common, respectively,

138 as well as 17 genes that were shared by all groups (Fig. 2b). These genes were enriched in

139 categories such as cell wall, hormone metabolism, phenylpropanoids and flavonoids, lignin

140 biosynthesis, peroxidases, TFs and oil biosynthesis. (Fig. 2c and d). It has been reported that

141 lignification increases in R2 and R3 of the elongation zone in soybean roots grown under water

142 deficit conditions, as well as in the equivalent regions of maize primary roots (23) . Therefore,

143 these results suggest that the set of 279 genes are likely involved in root elongation in the water

144 stress response and stress adaptation across different root regions. 
147 We analyzed the 279 water-stress responsive genes using MEME de novo motif discovery 148 algorithms and a total of 89 CREs were discovered in the promoter regions of these genes. 149 However, motif-searching tools can generate many false positive candidates, because transcription 150 factor binding sites are often short and typically contain some level of degeneracy in the binding 151 motif (24). We therefore performed multi-species phylogenetic footprinting (25) to identify 152 conserved noncoding sequences in soybean via a comparison of soybean with 11 dicot and one 153 monocot genome. We identified 49 of the 89 newly discovered motifs as conserved motifs, with a 154 false discovery rate of $<10 \%$ (Table S3). We used the STAMP tool (26) to compare the 49 155 conserved motifs with known cis-elements in the plant motif database PLACE (Fig. S1). Several 156 motifs, such as Root Drought-related motif (RDmotif) 18, 12, 5, 65, 22 and 93, showed significant 157 similarities (low E-values) to a G-box motif and ABA responsive elements (ABREs) 158 (PyACGTGG/TC). This result is consistent with the observations that ABA regulates the 159 expression of many genes under osmotic stress conditions and that ABRE is the major cis-element 160 for ABA-responsive genes (27). Furthermore, the RDmotif-14 is similar to DREB, another 161 drought-related motif (28).

\section{Traceback of core TFs ingroup using identified conserved motifs}

164 Extensive reprogramming of temporal and spatial transcription in response to stress occurs via the 165 interactions of TFs with a group of cis-regulatory motifs. The 49 conserved functional motifs 166 identified in this study could be used to explore how individual network nodes are wired within a 167 root developmental Gene Regulatory Network (GRN) in response to water stress and how 168 subcircuits are connected within the overall network hierarchy. However, the current cis-motif 169 datasets are generalized for TF families and cannot associate individual TFs with specific cognate 170 binding motifs. Thus, based on the finding that strong interactions usually occur between 171 individual subcircuits during development and that complex interactions occur among TFs 172 involved in multiple signaling pathways in response to stress $(7,12,20)$, we developed a strategy 173 to traceback a set of 49 conserved motif-associated core TFs that mutually regulate each other 174 and/or regulate themselves in response to water stress.

175 We reasoned that a core TF would have a cognate binding site motif, i.e., one of the 49 176 conserved motifs, in its own promoter and/or in the promoters of the other core TFs. Therefore, 
177 we identified 7954 soybean genes associated with the 49 conserved motifs using the multi-species 178 phylogenetic footprinting approach described above, with each of these conserved genes having 179 at least one of the 49 conserved motifs. Second, we performed GO enrichment analysis of the 7954 180 soybean genes to obtain a genome-wide overview of their functional roles (Fig. 3a). We classified 181 the conserved motifs and associated the genes into two groups based on GO annotation. In Group 182 I (GI), the conserved genes are primarily involved in plant growth and development. In Group II 183 (GII), the conserved genes are enriched in abiotic stress responses. Although these conserved 184 motifs play multifunctional roles in diverse biological processes, they might work together to process specific responses to water deficit in soybean roots. Interestingly, both GI and GII contain genes enriched in the category "response to endogenous stimulus". With the aim of revealing

187 important mediators of signaling between development and water stress responses, we focused on 188 a core set of TFs with two properties: 1) encoded by genes associated with both plant growth and 189 development-related motifs and abiotic stress-related motifs; and 2) responsive to both endogenous 190 and abiotic stimuli. Using these two criteria, we identified the set of 35 core TFs by overlapping 191 three groups of TFs (Fig. 3b): 1) GI-Endo: TFs annotated with the GO term "response to 192 endogenous stimulus" in GI; 2) GII-Endo: TFs annotated with the GO term "response to 193 endogenous stimulus" in GII; and 3) GII-Abiotic: TFs annotated with the GO term "response to abiotic stimulus" in GII.

We verified whether these 35 core TFs indeed respond to water stress in soybean primary root tissue. Since only 37,500 transcripts (approximately 23,787 genes) were examined in our microarray dataset, we introduced our recent RNA-seq-based dataset obtained by transcriptional profiling of soybean primary roots under water deficit (29). This primary root-specific transcriptome in soybean changes in response to very mild stress (VMS), mild stress (MS), severe 200 stress (SS) and recovery from severe stress after re-watering during a long period (21-days) under 201 various water-deficit conditions (Fig. S2a). Remarkably, the expression of 33 of the 35 core TFs 202 significantly differed in at least one of the soybean root-related transcriptome profiles, with 25 TFs 203 in common (Fig. 3b, Fig. S2b). Therefore, we named this set of 33 TFs the root drought-related 204 core TFs (rdcTFs) (Table S4).

The rdcTFs belong to several large multigene families, such as 206 Homeodomain/HOMEOBOX (HB), bHLH, basic leucine zipper (bZIP), APETALA2/ethylene207 responsive element binding protein (AP2/EREBP), NAC, Cys2-His2 zinc finger (C2H2), MYB 
and auxin response factor (ARF) (Table S4). Most rdcTFs contain at least five different conserved motifs (Fig. S2c), pointing to the combinatorial relationships of the 49 conserved motifs in the promoters of core TFs. For example, RDmotif-4 is often present in combination with other motifs, such as RDmotif-22 in GII and RDmotif-8, 24, 47 in GI (Fig. 3c), suggesting that genes containing

212 RDmotif-4 tend to respond to both abiotic stress and endogenous signals. We used this conserved 213 motif association principle to identify additional core TF candidates that have not yet been 214 assigned to GO terms but belong to our set of 279 root elongation-related water stress-responsive 215 genes. Three additional TFs associated with RDmotif-4 and abiotic stress-related RDmotif-22 were found, including two HAT22 homologs (Glyma01g40450 and Glyma17g16930) and one BEE (BR Enhanced Expression) gene Glyma07g10311. HAT22 is already known to be involved in an ABA-activated signaling pathway and the response to water deprivation (17). Interestingly, the BEE gene Glyma07g10311 was one of 17 common genes identified in the comparison of the 279 gene set with D5hR1, D48hR1 and D5hR2 (Fig. 2b).

\section{Co-functional core TF network regulating root growth in response to water stress}

Based on SoyBase genome annotation (http://www.soybase.org/genomeannotation/), we found that among the 33 rdcTFs and three additional core TF candidates, 27 TFs are known to be annotated with abiotic stress (e.g., water deprivation, osmotic, salt, cold, superoxide, peroxide, oxidative and heat stress) and 22 TFs are involved in the ABA signaling pathway, indicating that these core TFs regulate abiotic-stress responses in soybean roots (Table S5). These TFs are also responsive to multiple hormones and other signaling pathways, which suggest that these core TFs might orchestrate drought-related responses in soybean roots.

While much is known about the Arabidopsis genome, for other less well-studied plant species, such as soybean, the lack of knowledge regarding gene annotation and interactions severely limits network analysis using gene prioritization (30). Functional gene networks, such as

232 AraNet (31) and RiceNet (32), have proven to be useful for predicting gene functions and for 233 genetically dissecting plant traits. To investigate functional links among our core TFs involved in 234 multiple signaling pathways, we constructed a TF-TF co-functional network based on SoyNet 235 (www.inetbio.org/soynet), a database of co-functional networks of G. max genes, which enabled us to obtain the most comprehensive view of soybean pathway systems to date (33). We used the 
237 SoyNet to explore functional connections among the 33 rdcTFs and three additional core TFs, 238 finding 24 TFs that were wired to others in co-functional TF-TF network (Fig. S3). These 24 core

239 TFs are close together in the network, with an Area Under ROC Curve score of 0.8362 and p-value 240 3.7e-102, indicating that these core TFs are well predicted and associated with specific biological 241 processes (Table S6). After analyzing network statistics using Cytoscape (34), we ranked the TFs 242 based on their betweenness centrality (BC) to identify hub genes (Fig. S3 and Table S6). The BC 243 of a node reflects the amount of control that this node exerts over the interactions of other nodes

244 in the network, which helped us target hub TF genes that join different signaling pathways (dense 245 subnetworks), rather than TFs that lie inside a community (35). Network nodes at the intersections 246 of subcircuits are often controlled by signaling interactions (20).

247 In this TF-TF network, several homologs of core TFs, such as RVE7, RVE8, RAP2.4, BLH1, 248 IAA14, VNI2 and PIF4/PIF5 have been reported to function as mediators of signaling between a 249 developmental program and an environmental cue; these TF are involved in circadian, ABA, auxin, 250 ethylene and phytochrome signaling pathways for cell elongation, xylem formation, root 251 elongation and drought tolerance in Arabidopsis (36-38). To explore the dynamics of these core 252 TFs in response to water stress, we added the induction patterns for each TF using transcriptome 253 profiling data from soybean primary roots under various water-deficit conditions (Fig. S3 and Fig. 254 S2b). Several TF genes serving as hubs in the network, such as Glyma07g10311, Glyma10g05560, 255 Glyma08g47520 and Glyma20g35270, were induced by very mild stress, while most other TFs in 256 the network were induced by mild and/or severe stress conditions, implying that different core TFs

257 play different roles in regulating the response of soybean roots to water stress, depending on its 258 severity, or that they might function in succession as drought conditions progress from very mild 259 to severe through the integration of endogenous and external signals.

Mapping TF-DNA interactions using a high-throughput Y1H screen and the reconstruction 262 of a core TF network

263 We hypothesized that core TFs in the co-functional TF network play important roles in integrating 264 multiple abiotic stress and internal signals by regulating each other or themselves via binding to 265 conserved motifs located in their promoters. We therefore developed a quantitative motif/promoter 266 analysis platform to confirm the stress-response activity of the conserved motifs and their 267 associated promoters. We also performed a high-throughput $\mathrm{Y} 1 \mathrm{H}$ assay to measure TF-DNA 
interactions to verify the binding of the TFs.

We selected a hub gene, Glyma07g10311, a core TF from the 279 genes set, one of 17

genes that were shared by all groups of significantly differentially expressed genes (Fig. 2b), and also one of three additional TFs associated with RDmotif-4 tending to respond to both abiotic stress and endogenous signals, having the most connections with the other core TFs involved in different signaling pathways within the network, for further investigation: we named this gene BEE-like Root Growth regulator (BRG) (Fig. S3). Phylogenetic analysis showed that the combination of motif RDmotif-22 and RDmotif-4 located in the $B R G$ promoter is also found in most homologous genes across 13 species (Fig. 4a). RDmotif-22 is a G-box-type conserved motif that represents the conserved ABRE site gcCACGTGgc. Two phytochrome A-induced motifs, SORLIP1AT and SORLIP2AT are usually over-represented among root-specific genes (39). Another motif, CCAAT/ATTGG, was suggested binding site of NF-YB factors (40). To quantitatively characterize these motifs and their associated promoters, we constructed a single plasmid, pLL-QMP, harboring two reporter genes, firefly luciferase ( $L U C)$ and Renilla luciferase $(R E N)$ (Fig. 4b). A promoter region or motif repeats of interest was inserted before the minimal promoter to form a synthetic promoter to drive the expression of $L U C$ (Fig. S4). In this experiment, five different types of G-box motifs from GII and six representative motifs from GI were selected for analysis based on their inducibility by $100 \mu \mathrm{M} \mathrm{ABA}, 150 \mathrm{mM} \mathrm{NaCl} 2$ or $16 \%$ PEG treatment. Considering the degeneration of motif expression, each motif was designed based on the most frequent occurrence pattern in our root-specific 279 gene set (Fig. 4c, Table S7). The BRG promoter, which contains a motif named Gbox-I-gc, a representative RDmotif22 motif, was strongly induced by both salt and ABA. However, other types of G-boxes, such as Gbox-I-ac,

290 Gbox-I-tc, h-Gbox and their associated promoters PROM-Glymal1g27480, PROM291 Glyma06g03450 and PROM-Glyma12g33600, showed little or no induction by ABA or salt (Fig. 292 4d). In addition, selected representative motifs from Group I, such as dASE, dGA, dGT, dTG, 293 dGAGA and dACAC (related conserved motifs listed in Table S7), exhibited different levels of 294 induction by ABA, PEG and salt (Fig. 4e). Overall, this quantification pipeline provides a simple 295 method to determine the activities of various motifs/promoters in response to different stimuli. 
elongation microarray dataset, (Table S8). The final TF library contained $22 \mathrm{TF}$ families, including some well-known drought-related TFs or their homologs from the MYB, bZIP, AP2, NAC, bHLH and WRKY families (Fig. 5a). We used a conserved motif-centered Y1H platform to identify TFs that interact with the selected conserved motifs and their associated promoters. (Fig. 5b). In total, $70 \mathrm{TF}-\mathrm{DNA}$ interactions involving 12 different TFs were identified for the motif Gbox-I-gc group (Fig. 5c and Table S9). Moreover, 15 TF-DNA interactions involving 12 TFs were identified for the dASE motif (a representative of RDmotif-10), an ABA-independent osmotic stress-responsive motif (Fig. 5d). Notably, the Gbox-I-gc motif is preferentially bound by bZIP (Glyma06g04353), bHLH (Glyma10g28290) and NAC (Glyma06g21020, Glyma08g18470 and Glyma09g37050) TFs, whereas the dASE motif is bound by HB (Glyma02g04550, Glyma08g20170 and Glyma03g36070) TFs. In the Gbox-I-gc motif-centered network, six promoter fragments were classified into three groups based on their preferred binding TFs. Compared with the TFs that bind to mutant G-box (mGbox) and other negative promoters, the bZIP gene Glyma06g04353 (homolog of $A B F 2$ ), encoding a binding TF shared by motifs Gbox-I-gc, PROM-BRG and PROMGlyma04g04310, was characterized as a TF that targets the $B R G$ promoter. Thus, based on the binding specificity of PROM-BRG, Glyma06g04353 and the three other TFs were selected as the candidate regulators of $B R G$ for further network analysis. We named these genes based on their homology: ARG (Glyma06g04353, ABRE binding factor-like Root Growth regulator); $P R G$

317 (Glymalog28290, Phytochrome-interacting factor-like Root Growth regulator); NRG

318 (Glyma09g01650, NF-YB-like Root Growth regulator); and HRG (Glymal1g03850, HAT-like

Root Growth regulator).

After exploring the co-functional connections of the four candidate regulators with the other core TFs in SoyNet and integrating TF-DNA interactions into the co-functional TF-TF

322 network, we re-constructed a core TF network to visualize possible mutual regulation among core

323 TFs involved in multiple signaling pathways that function under water deficit in soybean roots

324 (Fig. 6, Table S10). The most highly ranked gene, $B R G$, is connected with $10 \mathrm{TFs}$. We discovered 325 that $A R G$ is the paralog of Glyma04g04170, and both genes respond to ABA stimulus and water 326 deprivation, while the two other genes that respond to water deficit, Glyma04g11290 and 327 Glym06g1101, homologs of $R A P 2.4$, are upregulated by cytokinin and ethylene. $H R G$ responds to $328 \mathrm{ABA}$ and auxin stimuli and is downregulated under salt and drought stress in soybean (41). $P R G$ 329 is a GA-responsive gene whose homolog, Glyma02g45150 functions in gravitropism responses to 
330 light. A homolog of RVE7 (Glyma16g34340) induced by ethylene stimulus and salt stress links $331 B R G$ with three other circadian rhythm-related genes, Glymal0g05560, Glyma07g05410 and Glyma16g01980. Moreover, Glyma08g47520 (homolog of VNI2, a transcriptional repressor of the master regulator VND7 during xylem vessel development), which responds to both ABA and BR, connects $B R G$ with two auxin-responsive genes and four $B L H 1$-like genes. Interestingly, these four BLH1-like genes respond to multiple hormones and abiotic stress, such as ABA, auxin, salicylic acid, ethylene, superoxide and salt stress. In addition, Glyma03g36070, one of the BLH1like genes in the network, has a dASE motif site in its own promoter, as do the 11 other core TFs. This observation suggests that Glyma03g36070 not only binds to the dASE site in its own gene and regulates its own expression, but it may also bind to dASE sites in many other core TFs in the network and regulate their expression. It has been reported that BLH1 itself enhances BLH1 expression (42), suggesting that the dASE motif site may participate in BLH1 self-regulation. RDmotif22 motif is associated with 10 core TFs besides $B R G$, which was already shown to be bound by ARG in our $\mathrm{Y} 1 \mathrm{H}$ assays. The link between $A R G$ and $N R G$ in the network suggests that these two TFs might function together by binding the RDmotif22 and CCAAT in the $B R G$ promoter. The detection of cis-regulatory motifs implies that the core TFs are heavily intertwined to ensure mutual regulation to coordinate the response to water deficit. $A R G, N R G$ and $P R G$ were upregulated under mild and severe water stress conditions in soybean roots, whereas the auxin signaling-related $H R G$ gene was upregulated under very mild water stress conditions and then downregulated, which is similar to the expression patterns of two IAA genes (Glyma20g35270 and Glyma08g22190) in the network. $B R G$ was upregulated under very mild water stress conditions and during the water recovery stage, a pattern unlike that of most of its associated genes in the network, suggesting that BRG might function as a repressor and/or be repressed by some of

353 its associated TFs through a tightly controlled network for the sequential activation of core TFs

354 under varying water deficit conditions. Four candidate regulators of BRG might also contribute to 355 the integration of multiple signaling pathways to help regulate root growth in response to water 356 stress.

359 To assess the acute $\mathrm{ABA}$ and osmotic stress responses of $B R G$, we generated hairy root lines harboring the promBRG::GFP reporter construct and measured reporter activity at $5 \mathrm{~h}$ and $48 \mathrm{~h}$ 
under $10 \mu \mathrm{M}, 50 \mu \mathrm{M}, 100 \mu \mathrm{M}$ ABA and PEG (-1.7 MPa) treatment. The reporter gene responded to $\mathrm{ABA}$, which was inversely correlated with $\mathrm{ABA}$ concentration and incubation time (Fig. 7a). In addition, we examined soybean root samples at 7 days after $10 \mu \mathrm{M}, 50 \mu \mathrm{M}$ and $100 \mu \mathrm{M}$ ABA treatment. $B R G$ levels significantly increased in response to $10 \mu \mathrm{M} A \mathrm{BA}$, followed by a decrease compared with the untreated control, whereas $A R G$ expression increased with increasing concentration of $\mathrm{ABA}$, which is in agreement the possible role of $\mathrm{ARG}$ as a repressor of $B R G$ expression (Fig. 7b). This hypothesis is also based on our finding that ARG bound to the $B R G$ promoter in $\mathrm{Y} 1 \mathrm{H}$ assays. We performed chromatin immunoprecipitation (ChIP) using transgenic hairy roots transformed with the $A R G$ minigene $(35 S:: A R G: G F P)$ after 3 days of dark treatment plus $100 \mu \mathrm{M}$ ABA until the expression of $B R G$ was repressed (Fig. 7c). We determined the enrichment of $B R G$ genomic regions after immunoprecipitation with an anti-GFP antibody by qPCR using primers covering the $B R G$ promoter, 5'UTR and CDS. Our results indicate that ARG associated with a region upstream of the transcription start site containing RDmotif-22 (CCACGTG) (Fig. 7d). Taken together, these results clearly demonstrate that ARG directly interacts with $B R G$ in vivo with or without $\mathrm{ABA}$ application.

To investigate the possible roles of selected key TFs in regulating root growth under water deficit, we generated transgenic soybean hairy roots overexpressing $B R G, A R G, N R G, H R G$ and $P R G$, as well as roots with loss-of-function due to RNA silencing (arg-si and brg-si) and roots expressing gene fused to the CRES repressor domain (ARG-cres and BRG-cres). We discovered that overexpressing $B R G$ and its four candidate regulators, had effect on root growth maintenance under ABA (Fig. S5a, b and g), NAA (Fig. S5f and g) and osmotic stress treatment (Fig. S5 c and g). Interestingly not all of four regulators appear to activate $B R G$ expression. Only overexpressing $H R G$ modestly upregulated $B R G$, whereas $B R G$ was downregulated in $A R G$-ox, $P R G$-ox and $N R G$ ox (Fig. S5j). BRG expression was reduced in $A R G$-ox and $A R G$-cres with or without ABA treatment, significantly increased in arg-si samples without ABA application, and slightly increased in the ARG deficient lines in response to ABA compared to the control (Fig. 8a). ARG expression in brg-si root lines was significantly higher than the control with or without ABA treatment, whereas $A R G$ was downregulated in both the $B R G$-ox and BRG-cres lines, indicating that BRG also negatively regulates the expression of $A R G$ (Fig. 8b).

We discovered that $B R G$-ox roots were significantly longer than empty vector control and $A R G$-ox roots under $10 \mu \mathrm{M} \mathrm{ABA}$ and PEG (-1.7 MPa) treatment, whereas $A R G$-ox roots were 
much longer than the control under ABA $50 \mu \mathrm{M}$ and $100 \mu \mathrm{M}$ treatment (Fig. 8c, d, e, f and h).

393 Interestingly, BRG-cres and brg-si primary roots did not lose the ability to maintain root growth, exhibiting some root growth under $10 \mu \mathrm{M}, 50 \mu \mathrm{M}$ and $100 \mu \mathrm{M}$ ABA treatment (Fig. 8c, d, e, f and h). This might be due to the presence of the other homologs or the strength of ARG in the absence of the repressive activity of BRG, since arg-brg-si roots did not sustain much more growth than control roots when treated with various concentrations of ABA or PEG treatment. Following pretreatment with PCZ for three days, only BRG-ox roots were hypersensitive to BL (0.5 nm), whereas the other roots were not (Fig. 8g and h). Overall, these results suggest that BRG and ARG are not genetically redundant in the regulation of root elongation. Moreover, their transcriptional activity might depend on the severity of water stress, and their antagonistic interplay, which occurs in an $\mathrm{ABA}$ dependent manner, indicates that the actions of BRG and $\mathrm{ARG}$ are not equally required throughout exposure to water stress signals.

ARG and BRG regulate a set of core TFs and have an ABA dose-dependent effect on darkgrown root growth

To investigate the roles of BRG and ARG in our core TF network, we analyzed the transcriptional activity of some selected core TFs when $A R G$ or $B R G$ was misregulated. $P R G$ expression in the BRG-ox, BRG-cres, ARG-cres, arg-si and arg-brg-si lines was significantly downregulated in response to $\mathrm{ABA}$ and more drastically enhanced in ARG-ox and brg-si lines with or without 100

$412 P R G$ (Fig. 8a, b and i). Therefore, we reasoned that ARG, BRG and PRG might jointly form a 413 feed-forward loop to adjust the responses to ABA. We found that NRG and HRG are involved in 414 these core $\mathrm{TF}$ interactions through direct binding to the $B R G$ promoter and that they regulate the 415 expression of $B R G, A R G$ and $P R G$. Together, we proposed a model describing the mutual 416 regulation among $A R G, B R G, P R G, N R G$ and $H R G$, which integrate multiple hormone pathways 417 with the phytochrome signaling pathway to sustain the growth of the underground root system 418 (Fig. 8j). We also observed significant differences in expression among the other core TF genes in 419 the network in BRG-ox, BRG-cres, ARG-cres, arg-si and arg-brg-si plants (Fig. S6). These 420 observations are in agreement with the notion that some core TFs have a direct effect on the 421 expression of the other core TFs, and they suggest that ARG and BRG together regulate these core 
422 TFs, which might coordinate water stress responses through the formation of extended cognitive

423 TF networks.

424 To study the roles of $\mathrm{ARG}$ and BRG in plant growth/development and in response to 425 drought conditions, we analyzed transgenic BRG-ox and ARG-ox Arabidopsis plants. The BRG-ox 426 and $A R G$-ox lines had larger leaves, higher biomass, longer primary root and total root length and 427 more root tips than wild-type (WT) Col-0 plants (Fig. S7 a and Fig. 9 a-d). Only the BRG-ox lines,

428 however, had larger floral organs and siliques than comparable WT and ARG-ox plants (Fig. S7 b 429 and c). In addition, ARG and NRG enhanced drought tolerance when overexpressed in Arabidopsis 430 (Fig. 9 e). We performed a root growth response assay in which WT, transgenic BRG-ox and $A R G$ 431 ox Arabidopsis plants were grown in long days for 3 days, followed by 7 days of growth on MS 432 medium supplemented with $10 \mu \mathrm{M}, 50 \mu \mathrm{M}$ ABA or PEG (-1.7 MPa) under continuous light or in 433 the dark. No significant difference was observed between WT, $A R G$-ox and BRG-ox plants under 434 continuous light, but the roots of dark-grown $A R G$-ox and BRG-ox plants were more tolerant to 435 ABA or PEG compared to dark-grown WT plants (Fig. $9 \mathrm{f}-\mathrm{g}$ ). We also found that only BEE1 436 expression was repressed in the dark, which corresponds to the presence of the ARG binding site 437 RDmoitf22 locating in the promoter of BEE1 (Fig. S7 d and e). Finally, to examine whether a 438 functional link also exists between the expression of $B R G$ and light regulation in soybean, we analyzed root growth in WT, BRG-ox, BRG-CRES and $b r g$-si soybean plants treated with $10 \mu \mathrm{M}$,

441 were more sensitive to ABA than WT, whereas dark-grown $B R G$-ox roots showed less inhibition 442 and continued growing in the presence of $10 \mu \mathrm{M}$ and $50 \mu \mathrm{M}$ ABA (Fig. $9 \mathrm{~h}$ and $\mathrm{i}$ ). Taken together, 443 these results suggest that regulons are conserved between $A R G$ and $B R G$, which have ABA 444 dependent effects on dark-grown roots, indicating that hormonal and light signaling pathways 445 utilize the same junctions to help plants adapt to water stress.

\section{DISCUSSION}

449 In this study, we developed an effective platform for identifying interconnected core TFs and 450 prioritized key regulators involved in root growth maintenance under water deficit in soybean. We 451 used four steps to identify and prioritize core TFs to help elucidate the regulation of root growth 452 under water deficit: 1) target a set of genes involved in root elongation in the water stress response 
and stress adaptation across different root regions; 2) identify a group of conserved functional motifs related to root growth regulation in response to water stress; 3) decompose genome-wide conserved motif-associated genes to trace back interconnected core TFs ingroup that respond to both endogenous and abiotic stimuli; and 4) combine co-functional TF-TF networks with TFDNA networks to prioritize key regulators. Using this platform, we succeeded in identifying a set of core TFs that are potentially important regulators of soybean root growth under water deficit conditions. Previous studies in Arabidopsis have shown that a number of homologs of these core TFs, e.g., ABF2, RVE7/8, RAP2.4, IAA14/IAA19, BLH1, PIF3/PIF4, HAT22, HAT2, NF-YB3, ZAT10 and VNI2, are involved in abiotic stress responses through mediating crosstalk among auxin, GA, BR, ABA, ethylene and phytochrome signaling. Therefore, we constructed a conserved core sensory TF network to explore their complex interconnected regulatory circuits by examining mutual cross-regulation among this group of TFs, which process signals to generate appropriate responses to water stress. Based on current and previous findings, we developed a model describing how root growth might be regulated under water deficit (Fig. 10).

The hormonal signaling mechanism that controls root growth in response to water availability is not yet fully elucidated. However, we know that ABA plays an important role in regulating stress-responsive genes, mainly through three bZIP TFs: AREB1/ABF2, AREB2/ABF4 and $\mathrm{ABF} 3$ (43). In our network, $A R G$ and another $A B F 2$-like gene (Glyma04g04170) were upregulated only in root tissue under water deficit, whereas other ABF genes including GmbZIPI (44) were upregulated in both root and shoot tissue (45). There are four BLH1 homologs in our core TF network. It has been reported that BLH1 interacted with another homeobox protein, KNAT3, and synergistically increased the ABA responses by binding to the promoter of $A B I 3$, which plays a role in auxin-induced lateral root formation (46). BLH1 also promotes its own

479 network analysis method can be used to associate specific groups of duplicate genes with an adaptive trait, which will help elucidate how the CREs in duplicate genes differ and why duplicate genes have different responses to stressful environments.

The auxin response acts downstream of $\mathrm{ABA}$ in regulating root elongation, which is 483 primarily dependent on altered Aux/IAA gene expression or altered gene expression in 
484

485

486

487

488

489

490

491

492

493

494

495

496

497

498

499

500

501

502

503

504

505

506

507

508

509

510

511

512

513

514

combination with additional factors (47). We also discovered the homologs of IAAI4/IAA19 in our network. GA promotes cell elongation largely by releasing the DELLA-mediated repression of PIF4, BZR1 and ARF6 (48). In particular, the existence of the BZR-ARF-PIF module explains how auxin, BR, GA, light and temperature coordinately regulate cell elongation in hypocotyls and likely other parts of the shoot in Arabidopsis (48). Based on our network, the BR-responsive gene $B R G$ appears to function as a signaling intermediary in multiple pathways, likely through interactions with homologous genes of ABF2, VNI2, RAP2.4, RVE7, PIF4/PIF5 and PIF3. PIF3 regulates hypocotyl length downstream of an auxin and ethylene cascade, whereas PIF4 and PIF5 regulate hypocotyl length upstream of this cascade (49). It remains to be elucidated whether the mechanisms underlying signaling integration involve a similar module in root tissue and how water stress signaling is integrated into this module.

Our present data suggest that BRG, ARG and PRG may achieve their roles in maintaining root growth through mediating crosstalk among BR, ABA and light signaling. ARG may play a major role in maintaining root growth in an ABA-dependent manner that leads to the downstream regulation of AUX/IAA factors to support auxin-related coordination between root elongation and lateral root development. BRG, on the other hand, may coordinate ABA-dependent and ABAindependent pathways through the regulation of two RAP2.4-like genes (Glymao6g11010 and Glyma04g11290), which might negatively regulate ethylene-mediated inhibition of root elongation (50). Our data suggest that ARG activates $P R G$ by repressing $B R G$, whereas $P R G$ and BRG negatively regulate each other in soybean root tissue. We discovered that a similar mechanism might function in soybean and Arabidopsis in which overexpressing $A R G$ represses BEE1 through binding to a conserved G-box motif in its promoter.

One notable regulatory mechanism is that HY5 and PIFs co-regulate photosynthetic genes at common G-box motifs, in conjunction with the circadian oscillator, to adjust the levels of rhythmic photosynthetic gene expression (51). In our network, ARG and PRG might act at a common G-box-type motif (RDmotif22) to regulate $B R G$ expression in response to water stress. Perhaps this dual control system works in conjunction with several circadian oscillator factors described in our network, including two LHY-like genes, two RVE7-like genes and one RVE8-like gene. CCA1 and LHY profoundly influence hypocotyl elongation and flowering time by regulating the expression of PIF4 and PIF5, respectively $(52,53)$. RVE7 acts primarily as a clock output gene and positively regulates the expression of an auxin biosynthetic gene (54). RVE8 affects light 
515 inhibition of hypocotyl elongation at low light intensities (55). The plant clock is organ specific,

516 which could be attributed to the different light inputs to the shoot versus root clocks (56). The

517 finding that ARG and BRG help maintain dark-grown root growth in response to ABA and osmotic

518 stress suggests that light has an additive, negative effect on root growth in response to these stresses,

519 which is similar to the finding that the illumination of roots alters salt and mannitol stress responses

520 (57). We therefore suggest that clock genes operate in conjunction with ARG, BRG, PRG and

521 other core TFs to adjust the levels of root growth in response to varying water deficit conditions

522 in the dark compared to their effect on the inhibition of shoot growth in the light.

523 One important feature of our platform is the identification of functionally conserved cis-

524 regulatory motifs and the traceback of these motif-associated core TFs as mediators of signaling

525 in response to both endogenous and abiotic stress stimuli. We suggest that water stress-responsive

526 gene expression in soybean roots is controlled by a core TF network consisting of mutually

527 regulated TFs that bind to the cognate motif sites in their own genes and regulate their own

528 expression and/or bind to the motif sites in many other core TF genes and regulate their expression.

Although both ARG and BRG help maintain root growth under water deficit conditions,

530 which core TF dominates this process might depend on the degree of water stress and/or $\mathrm{ABA}$

531 dose. BRG might play a more important role in rapid responses to water stress during early stages

532 of the water stress response by enhancing BR pathways and regulating ethylene production. During

533 long-term water stress, ARG might terminate this signaling under normal and very mild stress

534 conditions by repressing $B R G$ and the other factors involved, making a more "permanent decision"

535 to switch to a new cellular state or fate in response to more severe water stress signals. Future

536 studies should focus on determining the molecular mode of action of the core TF complexes in the

537 control of root growth and explore how these core TFs form complex feedback and feed-forward

538 loops. These loops might act as stress signal-sensitive delays or accelerators to regulate root growth

539 by efficiently coordinating cellular responses to very mild, mild and severe drought stress. The

540 strategies used in this study can be adopted to identify key genes and their interconnected networks

541 for other complex biological processes in plants or other species. Our core sensory TF network

542 lays the foundation for understanding the drought response in crops over long periods and should

543 provide guidance for further investigating the cell-type-specific transcriptional regulatory circuitry

544 that maintain root elongation but inhibit shoot growth under drought. Such studies might ultimately 
545 lead to the fine-tuning of cell-type-specific GRNs to improve crop tolerance and to avoid the

546 negative effects of drought on plant growth and yield.

\section{METHODS}

\section{Plant materials and growth conditions}

552 Soybean (Glycine max cv. Magellan) seeds were surface sterilized in 1\% NaClO solution for 2 $553 \mathrm{~min}$, rinsed in deionized water for $30 \mathrm{~min}$ and germinated between sheets of germination paper 554 moistened with a solution of $5 \mathrm{~mm} \mathrm{CaCl}_{2}$ and $5 \mathrm{~mm} \mathrm{Ca}(\mathrm{NO} 3)_{2}$ for $36 \mathrm{~h}$ at $29^{\circ} \mathrm{C}$ and near-saturation 555 humidity in the dark. Seedlings with primary roots approximately $15 \mathrm{~mm}$ long were transplanted 556 against the interior surfaces of Plexiglas cylinders $(14.5 \mathrm{~cm}$ diameter) filled with a $1: 1(\mathrm{v} / \mathrm{v})$ 557 mixture of vermiculite (no. 2A, Therm-ORock East Inc., New Eagle, PA, USA) and Turface 558 (Profile Products LLC, Buffalo Grove, IL, USA) at water potentials of -0.1 MPa (well-watered 559 treatment, moistened to the drip point) or $-1.6 \mathrm{MPa}$ (water-stressed treatment) according to 560 Yamaguchi et al. (2010). Three regions of soybean primary roots were identified based on their 561 growth under water stress treatment: region 1 (R1: 0-4 mm from the apex, including the root cap) 562 exhibits the same elongation rates under well-watered and water stress conditions; region 2 (R2: $5634-8 \mathrm{~mm}$ ) exhibits maximum elongation rates in well-watered soil, but progressive deceleration in 564 growth under water stress; and region $3(\mathrm{R} 3: 8-15 \mathrm{~mm})$ exhibits slow growth under well-watered 565 conditions and no growth under water stress. At $48 \mathrm{~h}$ time point (root tip water potential had 566 decreased to approximately -1.6 MPa by this time), R3 samples were collected under well-watered 567 conditions and there were no R3 samples under water stress conditions because root elongation 568 rates had been completely inhibited in R3. The root tips were sectioned into regions 1-2 (for both 569 water stressed and well-watered controls) at 5h, while 1-2 (water stressed) and 1-3 (well-watered 570 controls) at $48 \mathrm{~h}$, giving a total of nine samples per experiment. In each of three replicate 571 experiments, approximately 100 roots were harvested from the water-stressed treatment and from 572 the well-watered controls. The root samples were immediately frozen in liquid nitrogen and stored 573 at $-80^{\circ} \mathrm{C}$. 
576 Total RNA was isolated from root tissues using TRIZOL reagent (Invitrogen, Carlsbad, CA, USA).

577 Genomic DNA was eliminated by digesting the RNA with Turbo DNA-free DNaseI (Ambion)

578 according to the manufacturer's instructions. After DNaseI treatment, the RNA concentration was

579 determined and the quality checked using an Agilent 2100 Bioanalyzer (Agilent Technologies,

580 CA). Total RNA was used for Affymetrix Soybean Genome GeneChip hybridization and analysis

581 following the manufacturer's recommended protocols (Affymetrix, CA). cDNA synthesis, cRNA

582 amplification and the synthesis of sense-strand cDNAs were carried out using an Ambion WT

583 Expression kit. Hybridizations were conducted at the DNA Core Facility, University of Missouri

584 (http://biotech.rnet.missouri.edu/dnacore) following the standard Affymetrix procedures

585 (Affymetrix, Santa Clara, CA). The microarrays were scanned with a high resolution GeneChip

586 scanner. Scans from each microarray were analyzed with Affymetrix GeneChip Microarray Suite

587 version 5.0 software (MAS 5.0) in Expression Console version 1.1 of the Affymetrix GeneChip

588 Command Console suite. The microarray data sets used in the present study were deposited in the

589 Gene Expression Omnibus under accession number GSE102749

590 (http://www.ncbi.nlm.nih.gov/geo/query/acc.cgi?acc= GSE102749).

Microarray experiment and data analysis

593 The data files containing the probe set intensities (.cel files) were used for background correction 594 and normalization by the $\log 2$ scale GC-RMA procedure provided in GeneSpring Suite 11. Two595 way ANOVA was used to identify genes that were differentially expressed at the $5 \mathrm{~h}$ and $48 \mathrm{~h}$ time 596 points of five pairwise comparisons between WS and WW root regions R1, R2 and R3 with a False 597 Discovery Rate (FDR) of 0.05. Benjamini and Hochberg multiple testing corrections were used in 598 the Two-way ANOVA analysis. The differential expression levels of water-deficit-regulated genes 599 were clustered using DNA-Chip Analyzer (dChip) (Li and Wong, 2001). The functional 600 classification of differentially regulated genes was analyzed using MapMan (58). Enrichment of 601 each category was tested with Fisher's exact test (p-values $\leq 0.05$ ).

\section{Motif searching using MEME}

604 The 3-kb sequences upstream of the gene transcription start sites for all annotated soybean genes 605 (Phytozome v9.1) were collected from the same strand as the gene (in the same orientation). The 606 putative promoter regions for each of the clusters were written to individual files. Additionally, 
607 one file contained 279 randomly selected promoters, which were written to a separate file for use 608 as a representative promoter-negative set. Discriminative Motif Discovery (http://meme609 suite.org/tools/meme) was then performed using MEME V4.11. Position-Specific Priors (PSPs) 610 were derived for each cluster using clusters I, II and III as the positive set and the 279 randomly selected promoters as the negative set, with psp-gen. Using this discriminative method, all motifs

612 identified by MEME should be enriched in the clusters. MEME was then run using the -psp option,

613 -mod zoops (zero or one motif per sequence), specifying the maximum width of the motif as 15

$614 \mathrm{bp}$ and the minimum width as $6 \mathrm{bp}$ in both strands of sequences. In addition, motif sequences were 615 used to search each soybean promoter sequence. Counts of occurrence for the forward and reverse 616 complement of each motif were noted. Motif enrichment and significance levels were determined 617 using 1000 random gene sets, which were constructed by sampling without replacement using all 618 soybean genes. Thousand random samples of 279 genes were used to determine the mean 619 occurrence of each motif in the promoters of all genes. Observed counts for each motif found in 620 the 279-gene set were then compared to the mean. The probability that a motif is enriched was expressed as a p-value, and the p-value cutoff was 0.05 .

\section{Motif conservation analysis}

623 Motif phylogenetic conservation analysis was performed as previously described (25), with 624 Glycine max (Phytozome 9.1) as the query species and Arabidopsis thaliana (TAIR10), Carica papaya (Hawaii Agriculture Research Center), Theobroma cacao (Phytozome 9.1), Populus

626 trichocarpa (Phytozome 9.1), Prunus persica (Phytozome 9.1), Cucumis melo (Melonomics v3.5),

627 Eucalyptus glandiformis (Phytozome 9.1), Vitis vinifera (GeneScope v1), Solanum lycopersicum

628 (ITAG 2.3), Beta vulgaris (RefBeet 1.1), Oryza sativa (MSU RGAP 7) and Amborella trichopoda

629 (Amborella V1.0) as the comparator species from the PLAZA 3.0 database. For the alignment630 based approach, only the Sigma alignment tool (59) was used. The newly discovered motifs were 631 used as inputs for the comparative motif mapping approach and overlapped with the alignment632 based conserved non-coding sequences obtained for G. max. In the alignment-based approach, 633 pairwise alignments are generated between the query promoter (soybean putative promoter region: $6342 \mathrm{~kb}$ sequences upstream of gene transcription start) and the orthologous promoters. The algorithm 635 checks the significance of pairwise regions against a background model of 1000 randomly 636 generated orthologous groups for which the same process of alignment between the promoters is 
637 performed. A score is calculated based on both the length of the region and the number of species.

638 The p-value is calculated based on how many times the algorithm observes conservation scores in 639 the random groups that are as good as or better that the real conservation score from the original 640 orthologous group. All conserved motifs had a false discovery rate $<10 \%$.

\section{Motif similarity tree}

642 The newly discovered motifs were compared to motifs from the PLACE database using the 643 STAMP tool (26). Motif similarity was determined with the Pearson correlation coefficient and 644 aligned with the Ungapped Smith-Waterman algorithm, and a UPGMA tree was generated. Tree 645 visualization was performed using the FigTree tool (http://tree.bio.ed.ac.uk/software/figtree/).

\section{GO enrichment analysis of the conserved targets of newly discovered motifs}

647 GO annotations for G. max were obtained from the PLAZA 3.0 database (60). Of the 7954 target 648 genes obtained from the multispecies phylogenetic footprinting analysis, each gene had at least 649 one of the 49 previously identified conserved motifs. For each motif, the enrichment of conserved 650 motif target genes toward GO slim annotations (hypergeometric distribution + Bonferroni 651 correction) was determined. A heatmap was generated using Genesis (61), with a color gradient 652 that shows the P-values of the different enriched gene sets.

\section{Phylogenetic analysis}

655 To investigate their phylogenetic relationships, 20 BEE genes from 14 species were analyzed. 656 Protein sequences were analyzed by the neighbor joining (NJ) method with the genetic distance 657 calculated by MEGA v6.06 (62) (www.megasoftware.net/).

\section{Co-functional TF network construction}

660 The functional network of selected TFs is based on a genome-scale co-functional network of 661 soybean genes constructed using a machine learning approach with a Bayesian statistics 662 framework (33). To train various data that support the functional association between soybean 663 genes, a set of gold-standard gene pairs was generated by pairing two genes that share annotations 664 using the KEGG, MapMan or Soycyc pathway database. Various biological data, including the 665 co-citation of genes in research articles, co-expression, gene neighborhood, phylogenetic profiling 
and associalogs transferred from networks of the other species were also employed. The SoyNet (www.inetbio.org/soynet)-based method "find new members of a pathway" was used to explore functional connections among core TFs. Thirty-six TFs, including 33 rdcTFs and three core TFs candidates from 279 gene sets, were used as input genes. The SoyNet server was used to measure the retrieval rates of the 36 submitted TFs based on SoyNet connections among these TFs. The overall performance of the network for the retrieval of all core TFs was assessed based on receiver

672 operating characteristic analysis (63), which can be summarized as the area under the ROC curve 673 (64) score, indicating that core TFs for root growth regulation under water deficit are well 674 connected to each other based on SoyNet analysis. Twenty-four core TFs were ultimately found 675 to connect to each other in a co-functional TF-TF network, with the sum of LLS scores used to 676 rank their interactions. Network statistics analysis was applied in Cytoscape (34), and the TFs were 677 ranked based on their $\mathrm{BC}$ to current hub genes. The $\mathrm{BC}$ of a node reflects the amount of control 678 that this node exerts over the interactions of other nodes in the network (Yoon et al. 2006), which 679 helps target hub TFs that join communities (dense subnetworks) rather than TFs that lie inside a community. To integrate co-functional connections with TF-DNA interactions from Y1H, four candidate regulators ( $A R G, P R G, N R G$ and $H R G)$ of $\mathrm{BRG}$ were added in 36 core TFs list and were explored the co-functional connections of these genes in SoyNet. Then TF-DNA interactions were combined with all co-functional connections to create a total interactions list of core TFs for statistically analysis using Cytoscape to visualize possible mutual regulation among core TFs involved in multiple signaling pathways.

Construction of a Gateway-compatible library of soybean root-related water stressresponsive TFs

689 A representative TF library was constructed that included 214 root-related water stress-responsive TFs in 22 TF families. These drought-related TFs were selected based on a >2-fold change at least in one of five comparisons from soybean root elongation microarray data in response to water 692 deficit. Soybean TF coding sequences were amplified by PCR and cloned into the pDONR221 693 Gateway donor vector (Life Technologies). All clones included in the collection (214 TFs) were 694 sequence validated (Table S8). Each TF was transferred into a Y1H-compatible destination 695 plasmid (pDEST22) carrying the GAL4-Activation Domain (GAL4_AD) located at the TF 
697 (Invitrogen). TFs cloned in pDEST22 were thus expressed in yeast as C-terminal fusions to 698 GAL4_AD. After destination plasmid validation by gene-specific colony PCR and sequencing, 699214 clones were successfully cloned into pDEST22. The pDEST22-TF collection was arrayed in 700 three 96-well plates.

701

Bait construction and yeast one-hybrid assay

703 A total of 214 E. coli strains harboring different soybean TFs were arrayed in three 96-well plates, and plasmids were prepared using the Zymore plasmid purification DNA system according to the manufacturer's recommendations. The oligonucleotides used to amplify motif repeats and promoter fragments ( $500 \mathrm{bp}$ ) for mGbox, Gbox-I-gc, dASE, mdASE, PROM-BRG, PROMGlyma05g26930, PROM-Glyma04g04310, PROM-Glyma06g03450, PROM-Glyma02g15780 and PROM-Glyma08g29100 are described in Supplemental Table S14. These bait DNAs were subcloned into pENTR/TOPO-D (Gateway; Invitrogen), followed by pGLAC recombined with the reporter gene $\beta$-galactosidase $(\mathrm{Gal})$. The pGLAC recombinants were transformed into yeast strain Y4271 and screened against the root drought stress-related TF library using the Y1H protocol, as previously described (65). The DNA bait strains were tested for self-activation prior to screening by transforming them with prey vectors. pDEST22 empty vector was used as a negative control. The transformants were grown on SD -Trp plates (Clontech, 2\% agar) for selection. Positive interactions were visually identified by the yellow color of the yeast cells caused by the cleavage of ortho-nitrophenyl from colorless ortho-nitrophenyl- $\beta$-D-galactoside by $\beta$ galactosidase. Each TF was tested in quadruplicate. The first screen was applied against the whole

718 TF library with replicates. All interacting TFs were assembled into a small interaction library for 719 the second screen. The second screen was applied with replicates in the same plate. Each positive

720 TF clone was sequenced to reconfirm its identity.

\section{Dual-Luc assay-based quantification of the stress inducibility of conserved motifs/promoters}

723 A vector system was created to generate a single vector with the CaMV $35 \mathrm{~S}$ mini promoter $(35 S)$

724 fused to the firefly luciferase reporter gene and $35 S$ fused to the Renilla luciferase reporter gene.

725 The constitutively expressed Renilla gene served as a control to normalize for transformation 726 efficiency. This system included one destination vector, pLL-QMP, and one entry vector, pLL$727 \mathrm{P} / \mathrm{M}$, with Gateway Technology (Invitrogen) used to clone the promoter fragment/motif repeats 
728 (Figure S3). The 3 kb pRTL2-Renilla HindIII digested fragment was inserted into SacI-digested 729 pFLASH to create pFLASH-FR with both firefly LUC and Renilla luciferase (REN) genes. 730 HindIII-digested pFLASH-FR was ligated with the HindIII-digested PCR fragment Mini35S to 731 yield pLL-QMP (Figure S4). Target motif repeats/promoter fragments were amplified from $G$. $732 \max$ genomic DNA using the appropriate primers with attB1 and attB2 sites. Each amplified 733 fragment was cloned into a pDONR-P1-P2 vector by performing BP reactions to produce pLL734 P/M. Finally, the fully functional expression vector pLL-QMP-PM was generated by Gateway LR 735 cloning (Figure S4).

Transgenic soybean hairy roots were then generated (detailed below) using each final pLL-

737 QMP-PM construct. Agrobacterium rhizogenes strain K599 was transformed with the pLL-QMP-

738 PM construct by electroporation. The bacterial solution was drawn into a syringe and injected three 739 times into the cotyledon node and the upper hypocotyls. Infected seedlings were transferred into 740 pots. The plants were maintained in a growth chamber under a $12 \mathrm{~h}$ light $/ 12 \mathrm{~h}$ dark cycle at $28^{\circ} \mathrm{C}$ 741 for 2-3 weeks, at which time the hairy roots were approximately 5-10 cm long. Hairy root samples 742 were harvested $6 \mathrm{~h}$ after treatment with $100 \mu \mathrm{M} \mathrm{ABA}, 150 \mathrm{mM} \mathrm{NaCl} 2$ or $16 \%$ PEG and assayed 743 for luciferase activity using the Dual-Luciferase Reporter Assay System (Promega) according to 744 the manufacturer's instructions. Approximately $100 \mathrm{mg}$ of tissue was frozen in liquid $\mathrm{N}$ and 745 homogenized using a Retsch Mixer Mill MM400 for 1 min at $30 \mathrm{~Hz}$. The ground tissue was thawed 746 in lysis buffer (0.1 M HEPES, pH 7.8, $1 \%$ Triton X-100, $1 \mathrm{mM} \mathrm{CaCl}_{2}$ and $\left.1 \mathrm{mM} \mathrm{MgCl}_{2}\right)$ at $25^{\circ} \mathrm{C}$ 747 for $15 \mathrm{~min}$. Luciferase Assay Reagent II $(50 \mu \mathrm{L})$ was added to $10 \mu \mathrm{L}$ aliquots of the lysates to 748 measure firefly luciferase activity using a Spectra Max M5/M5e plate reader to measure total light 749 emission after a $1000 \mathrm{~ms}$ integration time. Firefly luciferase activity was quenched with $50 \mu \mathrm{L}$ of 750 Stop \& Glo Reagent, which contains Renilla luciferin substrate, and total light emission was 751 measured at a $100 \mathrm{~ms}$ integration time. An expression vector containing part of the coding 752 sequence of the $\beta$-glucuronidase reporter gene rather than a TF gene was used to measure baseline 753 firefly luciferase activity. To estimate the relative affinity of the TFs for each promoter fragment, 754 five biological replicates of TF-expressing vectors were compared to the average results for the 755 GUS expression vector. Firefly luciferase activity was divided by Renilla luciferase activity to 756 normalize the transformation efficiency of each hairy root sample. The relative binding of the TFs 757 to promoter bait sequences was determined relative to the control using two-way ANOVA + Tukey 758 HSD. 
Generation of transgenic soybean hairy roots

761 Soybean line Williams 82 was used in this study. Sterile soybean seeds were germinated and grown

762 in sterilized vermiculite for 7 days in a growth chamber at $25^{\circ} \mathrm{C}$ under fluorescent white light with

763 a 16/8 h light/dark cycle. Transgenic soybean hairy roots were generated as previously described

764 (66) with some modifications. Agrobacterium rhizogenes strain K599 was transformed with each

765 individual construct by electroporation. Cotyledons from geminated soybean seedlings were

766 inoculated with the bacterial culture to induce hairy roots, as previously described (66).

Fourteen days post-inoculation, transgenic hairy roots were identified based on growth on

768 hygromycin. The roots were isolated and removed from the cotyledons 18-25 days post-

769 inoculation and cultured on $1 / 2$ MS solid medium (3\% sucrose, $0.6 \%$ agar) plus carbenicillin (500

$770 \mathrm{mg} / \mathrm{L}$ ) in the dark at $26^{\circ} \mathrm{C}$. After 2-3 weeks, 3-4 cm long lateral roots or the primary root tip were

771 cut and subcultured on $1 / 2$ MS solid medium plus carbenicillin (500 mg/L). After subculturing 3-4

772 times on $1 / 2$ MS solid medium containing carbenicillin, transgenic roots were used immediately,

773 frozen or maintained on $1 / 2$ MS solid medium (without carbenicillin) for treatment. The

774 concentrations of the treatments were as follows: $(10,50,100) \mu \mathrm{M} \mathrm{ABA}, 50 \mu \mathrm{M}$ naphthaleneacetic

775 acid (NAA), (5, 0.5, 0.05) $\mu \mathrm{M}$ BL and $10 \mu \mathrm{M}$ PCZ. Stocks were made in $80 \%$ ethanol at identical

776 dilutions except for NAA, for which the stock was dissolved in sodium hydroxide, with mock

777 treatment performed using an equal amount of solvent alone. For the PEG, ABA, BL, PCZ and

778 NAA treatments, transgenic hairy roots were incubated in the dark on PEG-infused plates $(-1.7$

$779 \mathrm{MPa})(67,68)$ or $1 / 2 \mathrm{MS}$ solid medium containing $100 \mu \mathrm{M}$ ABA, $50 \mu \mathrm{M}$ naphthaleneacetic acid

780 (NAA), $(5,0.5,0.05) \mathrm{nM}$ BL or $10 \mu \mathrm{M}$ PCZ and measured 7 days later ( $\mathrm{n}=12-15$, three repeats).

781 Statistical analysis for this study was carried out with R (version 3.2.4). For each analysis of effects

782 of different genotypes on hormone/osmotic stress response, two-way ANOVA followed by Tukey

783 HSD multiple comparison procedure were used to detect significant differences between mock

784 control and dose of hormone/osmotic stress treatments associated with genotypes and primary root

785 length. The linear model is $\mathrm{y}=$ intercept+ hormone/osmotic stress + genotype + interaction between

786 stress and genotype +residual error. We first performed two-way ANOVA which not only assess

787 the independent impact of the two factors but also can assess the interaction of the two factors.

788 When the interaction term was found to be significant, we concluded that genotype affected 
hormone/osmotic stress response. $P$-value associated with particular levels of hormone/osmotic stress treatment were adjusted for multiple comparisons using Turkey HSD method.

\section{Quantitative RT-PCR analysis}

793 Total RNA was extracted from root samples collected in the morning using an RNeasy kit (Qiagen).

794 Genomic DNA was removed using an RNase-free DNase set (Cat\# 79254, Qiagen). The cDNA

795 was synthesized via treatment with reverse transcriptase and oligo(dT) primer (SuperScript III

796 First-Strand Synthesis System; Invitrogen). Quantitative RT-PCR was performed on an

797 ABI7900HT detection system (Life Technologies) using Maxima SYBR Green/ROX qPCR

798 Master Mix (2X) (Cat\# K0223, Thermo, USA) following the manufacturer's protocol. The primer

799 sets used for qRT-PCR are listed in Table S14. The comparative Ct method for quantification was

800 used to quantify the relative expression levels of specific genes (69). Gene expression was 801 measured in at least three biological replicates with three technical replicates. Significance relative 802 to control was determined by two-tailed Welch's $t$-test.

\section{Molecular cloning and constructs}

805 The $2 \mathrm{~kb} B R G$ promoter of was subcloned in pENTR/TOPO-D and fused to GFP in the Gateway806 compatible destination vector pMDC107 for soybean hairy root transformation. The $A R G, B R G$,

$807 H R G, N R G$ and $P R G$ overexpression constructs were generated by subcloning in pDONR221, 808 followed by cloning in pMDC32. For the ChIP construct, $A R G$ was subcloned in pDONR221 and 809 then fused to GFP in the pMDC83 vector. The Arg-si and Brg-si vectors were generated by 810 subcloning synthetic fragments (Table S14) in pENTR/TOPO-D, followed by cloning in the 811 destination vector pANDA (RNAi construct). The ARG-cres and BRG-cres vectors were 812 subcloned in pDONR221 and fused to the CRES domain in pB7WG2-CRES. The primers used 813 are listed in Table S14.

\section{Confocal laser-scanning microscopy}

816 Confocal laser-scanning microscopy was carried out on a Leica TCP SP8. Excitation was 817 performed using a 488-nm laser for GFP. Cell walls were stained with propidium iodide as 818 previously described (69). Transgenic hairy root tips (PROM-BRG::GFP) used for scanning were 
819 placed onto PEG-infused plates $(-1.7 \mathrm{MPa})(67,68)$ or MS solid medium containing $10 \mu \mathrm{M}, 50$

$820 \mu \mathrm{M}$ or $100 \mu \mathrm{M} \mathrm{ABA}$ at $5 \mathrm{~h}$ or $48 \mathrm{~h}$.

823 Chromatin immunoprecipitation was conducted as previously described (65) Approximately $5 \mathrm{~g}$ 824 (fresh weight) of root tissue (35S::GFP and 35S::ARG::GFP) on day 3 with/without ABA (100 $825 \mu \mathrm{M})$ treatment was harvested and crosslinked for 15 min under a vacuum in crosslinking buffer 826 (10 mM Tris, pH 8.0, $1 \mathrm{mM}$ EDTA, $250 \mathrm{mM}$ sucrose, $1 \mathrm{mM}$ PMSF and 1\% formaldehyde). 827 Technical replicates containing approximately $1.5 \mathrm{mg}$ DNA were resuspended in $800 \mu \mathrm{L}$ SII buffer, 828 incubated with $2 \mu \mathrm{g}$ anti-GFP antibodies (ab290, Abcam) bound to Protein G Dynabeads 829 (Invitrogen) for $1.5 \mathrm{~h}$ at $4^{\circ} \mathrm{C}$ and washed five times with SII buffer. Chromatin was eluted from 830 the beads twice at $65^{\circ} \mathrm{C}$ with Stop buffer $(20 \mathrm{mM}$ Tris-HCl, pH 8.0, $100 \mathrm{mM} \mathrm{NaCl}, 20 \mathrm{mM}$ EDTA 831 and 1\% SDS). RNase- and DNase-free glycogen $(2 \mu \mathrm{g})$ (Boehringer Mannheim) was added to the 832 input and eluted chromatin, after which they were incubated with DNase- and RNase-free 833 proteinase $\mathrm{K}$ (Invitrogen) at $65^{\circ} \mathrm{C}$ overnight and treated with $2 \mu \mathrm{g}$ RNase A (Qiagen) for $1 \mathrm{~h}$ at $83437^{\circ} \mathrm{C}$. DNA was purified using a Qiagen PCR Purification kit and resuspended in $100 \mu \mathrm{L} \mathrm{H}_{2} 0$. 835 Quantitative PCR of the technical replicates was performed using a QuantiFast SYBR Green PCR 836 kit (Qiagen) with the following cycling conditions: 2 min at $95^{\circ} \mathrm{C}$, followed by 40 cycles of $15 \mathrm{~s}$ 837 at $95^{\circ} \mathrm{C}, 15 \mathrm{~s}$ at $55^{\circ} \mathrm{C}$ and $20 \mathrm{~s}$ at $68^{\circ} \mathrm{C}$. The primers used in this study are listed in Table S14. The 838 results were normalized to the input DNA using the following equation: $100 \times 2^{\text {(Ct input-Ct ChIP) }}$.

\section{Constructs and generation of transgenic Arabidopsis plants}

841 Full-length cDNA of the selected genes $A R G, P R G, H R G, N R G$ and $B R G$ was amplified using the

842 primers listed in Table S14. The amplified fragments were introduced into the pDONR221 vector 843 (Invitrogen) and cloned into pMDC32 by LR reactions (Invitrogen). The construct was 844 electroporated into Agrobacterium tumefaciens GV3101 and transformed into Col-0. Sterilized 845 Arabidopsis seeds were grown on $1 / 2$ MS medium with $1 \%$ sucrose solidified with $0.3 \%$ Phytagel 846 under a $16 \mathrm{~h} / 8 \mathrm{~h}$ light/dark cycle at $22^{\circ} \mathrm{C}$. For drought stress treatment, 14-day-old T3 transgenic 847 Arabidopsis plants were subjected to control (well-watered) and drought stress (withholding water) 848 conditions for an additional 12 days. Two transformation events were used for the $A R G$-ox and $849 N R G$-ox plants, respectively. More than three pots were prepared for each transformation event, 
850 with 25 plants per pot, and all pots were rotated daily during drought stress treatment to minimize

851 environmental effects. For the root elongation assays, the germinated seedlings were transferred

852 to medium in square Petri dishes and grown vertically in long days for 7 days. For the PEG and

853 ABA treatments, WT (Col-0), ARG-ox and BRG-ox homozygous Arabidopsis plants were grown

854 in long days for 3 days, followed by 7 days of growth on $1 / 2$ MS medium (1\% sucrose, $0.3 \%$

855 Phytagel) supplemented with $10 \mu \mathrm{M}$ ABA, $50 \mu \mathrm{M}$ ABA or PEG (-1.7 Mpa) under continuous light

856 or in the dark. At least 18-24 seedlings were measured for each genotype in each set of experiments.

857 Total root length and root tip number were measured using Rhizo software (70). Primary root

858 length was measured from scanned images using ImageJ software. Statistical analysis was

859 performed in R (version 3.2.4) using two-way ANOVA + Tukey HSD for multiple comparisons

860 as described in Generation of transgenic soybean hairy roots

861

\section{ABBREVIATIONS}

ABA: abscisic acid

AUX: auxin

GA: gibberellic acid

ETH: ethylene

SA: salicylic acid

JA: jasmonic acid

CK: cytokinin

BR: brassinosteroid

bZIP: basic leucine zipper 
AP2/EREBP: APETALA2/ethylene-responsive element binding protein

$\mathrm{C} 2 \mathrm{H} 2$ : Cys2-His2 zinc finger

ARF: auxin response factor

VMS: very mild stress

MS: mild stress

SS: severe stress

CREs: cis-regulatory elements

\section{DECLARATIONS}

\section{Ethics approval and consent to participate}

893 Not applicable

\section{Consent for publication}

896 Not applicable

\section{Availability of data and materials}

898 All data generated or analysed during this study are included in this published article [and its

899 supplementary information files]. The microarray data sets used in this study were deposited in

900 the Gene Expression Omnibus under the accession number of GSE102749

901 (http://www.ncbi.nlm.nih.gov/geo/query/acc.cgi?acc= GSE102749 ). The following secure token

902 has been created to allow review of record GSE102749 while it remains in private status:

903 gjgvekembzmdzcj.

\section{Competing interests}

905 The authors declare that they have no competing interests

\section{Funding}

907 This work was funded by a United Soybean Board (project\# 1204) grant to H.N. 


\section{Authors' contributions}

909 L.L. designed the research. J.V.d.V., K.V., Y.A. and L.L. performed conserved motif analysis. N.

$910 \mathrm{~N}$ and L.L. contributed phenotype analysis. L.L., J.W., L.S., and W.C. performed the Dual-Luc

911 assay. L.L., and G.P. performed the hairy root transformation. G.P. and L.L. contributed the

912 Arabidopsis transformation. S.P., L.S., and M.M. performed the RNA-seq experiments and B.V.

913 performed the microarray experiments. E.K., I.L. and L.L. contributed the co-functional TF-TF

914 network. L.L. and G.P. constructed the TF library. L.L. performed Y1H assay and constructed TF-

915 DNA networks. C.Z., G.K., T.J., L.L. and D.X. contributed bioinformatics analyses. L.L. wrote

916 the article. H.N. and K.V. critically reviewed the manuscript.

918 Acknowledgements

919 This work was funded by a United Soybean Board (project\# 1204) grant to H.N. We thank Dr.

920 Steve Kay for the pFLASH vector, Dr. Zhiyong Wang and Tao Wang for the comments and

921 suggestions. K.V. acknowledges the Multidisciplinary Research Partnership "Bioinformatics:

922 from nucleotides to networks" Project (no. 01MR0310W) of Ghent University. J.V.d.V is indebted

923 to the Agency for Innovation by Science and Technology (IWT) in Flanders for a pre-doctoral

924 fellowship.

925

\section{REFERENCES}

927 1. Koevoets IT, Venema JH, Elzenga JT, \& Testerink C (2016) Roots Withstanding their

928 Environment: Exploiting Root System Architecture Responses to Abiotic Stress to

929 Improve Crop Tolerance. Front Plant Sci 7:1335.

9302 2. Sharp RE, et al. (2004) Root growth maintenance during water deficits: physiology to 931 functional genomics. J Exp Bot 55(407):2343-2351.

932 3. Rowe JH, Topping JF, Liu J, \& Lindsey K (2016) Abscisic acid regulates root growth

933 under osmotic stress conditions via an interacting hormonal network with cytokinin,

934 ethylene and auxin. New Phytol 211(1):225-239.

935 4. Zhu JK (2016) Abiotic Stress Signaling and Responses in Plants. Cell 167(2):313-324.

936 5. Jaillais Y \& Chory J (2010) Unraveling the paradoxes of plant hormone signaling 937 integration. Nat Struct Mol Biol 17(6):642-645. 
938 6. Fujita Y, Fujita M, Shinozaki K, \& Yamaguchi-Shinozaki K (2011) ABA-mediated

939 transcriptional regulation in response to osmotic stress in plants. J Plant Res 124(4):509-

$940 \quad 525$.

941 7. Nakashima K, Yamaguchi-Shinozaki K, \& Shinozaki K (2014) The transcriptional

942 regulatory network in the drought response and its crosstalk in abiotic stress responses

943 including drought, cold, and heat. Front Plant Sci 5:170.

944 8. Singh D \& Laxmi A (2015) Transcriptional regulation of drought response: a tortuous

945 network of transcriptional factors. Front Plant Sci 6:895.

9469 9. Nguyen D, Rieu I, Mariani C, \& van Dam NM (2016) How plants handle multiple

947 stresses: hormonal interactions underlying responses to abiotic stress and insect

948 herbivory. Plant Mol Biol 91(6):727-740.

949 10. Kim JS, et al. (2011) An ABRE promoter sequence is involved in osmotic stress-

950 responsive expression of the DREB2A gene, which encodes a transcription factor

951 regulating drought-inducible genes in Arabidopsis. Plant Cell Physiol 52(12):2136-2146.

952 11. Lee SJ, et al. (2010) DREB2C interacts with ABF2, a bZIP protein regulating abscisic

953 acid-responsive gene expression, and its overexpression affects abscisic acid sensitivity.

$954 \quad$ Plant Physiol 153(2):716-727.

955 12. Joshi R, et al. (2016) Transcription Factors and Plants Response to Drought Stress:

956 Current Understanding and Future Directions. Front Plant Sci 7:1029.

957 13. Nakashima K, Takasaki H, Mizoi J, Shinozaki K, \& Yamaguchi-Shinozaki K (2012)

958 NAC transcription factors in plant abiotic stress responses. Biochim Biophys Acta

959 1819(2):97-103.

960 14. Sakuraba Y, Kim YS, Han SH, Lee BD, \& Paek NC (2015) The Arabidopsis

961 Transcription Factor NAC016 Promotes Drought Stress Responses by Repressing

962 AREB1 Transcription through a Trifurcate Feed-Forward Regulatory Loop Involving

$963 \quad$ NAP. Plant Cell 27(6):1771-1787.

964 15. Cheng MC, Liao PM, Kuo WW, \& Lin TP (2013) The Arabidopsis ETHYLENE

965 RESPONSE FACTOR1 regulates abiotic stress-responsive gene expression by binding to

966 different cis-acting elements in response to different stress signals. Plant Physiol

967 162(3):1566-1582. 
968 16. Denyer T, et al. (2019) Spatiotemporal Developmental Trajectories in the Arabidopsis

969 Root Revealed Using High-Throughput Single-Cell RNA Sequencing. Dev Cell

$970 \quad 48(6): 840-852$ e845.

971 17. Song L, et al. (2016) A transcription factor hierarchy defines an environmental stress

972 response network. Science 354(6312).

973 18. Schmutz J, et al. (2010) Genome sequence of the palaeopolyploid soybean. Nature

974 463(7278):178-183.

975 19. Niwa H (2018) The principles that govern transcription factor network functions in stem 976 cells. Development 145(6).

977 20. Peter IS \& Davidson EH (2017) Assessing regulatory information in developmental gene 978 regulatory networks. Proc Natl Acad Sci U S A 114(23):5862-5869.

979 21. Schaffter SW \& Schulman R (2019) Building in vitro transcriptional regulatory networks 980 by successively integrating multiple functional circuit modules. Nat Chem 11(9):829-838.

981 22. O'Malley RC, et al. (2016) Cistrome and Epicistrome Features Shape the Regulatory 982 DNA Landscape. Cell 165(5):1280-1292.

983 23. Yamaguchi M, et al. (2010) Regulation of growth response to water stress in the soybean primary root. I. Proteomic analysis reveals region-specific regulation of phenylpropanoid metabolism and control of free iron in the elongation zone. Plant Cell Environ 33(2):223-

987 24. Tompa M, et al. (2005) Assessing computational tools for the discovery of transcription factor binding sites. Nat Biotechnol 23(1):137-144.

25. Van de Velde J, Heyndrickx KS, \& Vandepoele K (2014) Inference of transcriptional

992 26. Mahony S \& Benos PV (2007) STAMP: a web tool for exploring DNA-binding motif 993 similarities. Nucleic Acids Res 35(Web Server issue):W253-258.

994 27. Maruyama K, et al. (2012) Identification of cis-acting promoter elements in cold- and 995

997 28. Mizoi J, Shinozaki K, \& Yamaguchi-Shinozaki K (2012) AP2/ERF family transcription 998 factors in plant abiotic stress responses. Biochim Biophys Acta 1819(2):86-96. 
999 29. Song L, et al. (2016) Genome-wide transcriptome analysis of soybean primary root under varying water-deficit conditions. BMC Genomics 17(1):57.

30. Serin EA, Nijveen H, Hilhorst HW, \& Ligterink W (2016) Learning from Co-expression

1002 Networks: Possibilities and Challenges. Front Plant Sci 7:444.

1003

31. Lee I, Ambaru B, Thakkar P, Marcotte EM, \& Rhee SY (2010) Rational association of 1004 1005

32. Lee T, et al. (2015) RiceNet v2: an improved network prioritization server for rice genes.

33. Kim E, Hwang S, \& Lee I (2016) SoyNet: a database of co-functional networks for soybean Glycine max. Nucleic Acids Res.

1010

34. Smoot ME, Ono K, Ruscheinski J, Wang PL, \& Ideker T (2011) Cytoscape 2.8: new features for data integration and network visualization. Bioinformatics 27(3):431-432.

35. Yoon J, Blumer A, \& Lee K (2006) An algorithm for modularity analysis of directed and weighted biological networks based on edge-betweenness centrality. Bioinformatics 22(24):3106-3108.

36. Castillon A, Shen H, \& Huq E (2007) Phytochrome Interacting Factors: central players in phytochrome-mediated light signaling networks. Trends Plant Sci 12(11):514-521.

37. Lucyshyn D \& Wigge PA (2009) Plant development: PIF4 integrates diverse environmental signals. Curr Biol 19(6):R265-266.

38. Rawat R, et al. (2009) REVEILLE1, a Myb-like transcription factor, integrates the circadian clock and auxin pathways. Proc Natl Acad Sci U S A 106(39):16883-16888.

40. Gusmaroli G, Tonelli C, \& Mantovani R (2001) Regulation of the CCAAT-Binding NFY subunits in Arabidopsis thaliana. Gene 264(2):173-185. expression profiling under salinity and drought treatments. Plos One 9(2):e87156.

1028 42. Staneloni RJ, et al. (2009) Bell-like homeodomain selectively regulates the highirradiance response of phytochrome A. Proc Natl Acad Sci U S A 106(32):13624-13629. 
1030 43. Fujita Y, Yoshida T, \& Yamaguchi-Shinozaki K (2013) Pivotal role of the AREB/ABFSnRK2 pathway in ABRE-mediated transcription in response to osmotic stress in plants. Physiol Plant 147(1):15-27.

44. Gao SQ, et al. (2011) The soybean GmbZIP1 transcription factor enhances multiple abiotic stress tolerances in transgenic plants. Plant Mol Biol 75(6):537-553.

45. Song L, et al. (2016) Genome-wide transcriptome analysis of soybean primary root under varying water-deficit conditions. BMC Genomics 17:57.

46. Kim D, et al. (2013) BLH1 and KNAT3 modulate ABA responses during germination and early seedling development in Arabidopsis. Plant J 75(5):755-766.

47. Belin C, Megies C, Hauserova E, \& Lopez-Molina L (2009) Abscisic Acid Represses Growth of the Arabidopsis Embryonic Axis after Germination by Enhancing Auxin Signaling. Plant Cell 21(8):2253-2268.

48. Oh E, et al. (2014) Cell elongation is regulated through a central circuit of interacting transcription factors in the Arabidopsis hypocotyl. Elife 3.

49. Bours R, Kohlen W, Bouwmeester HJ, \& van der Krol A (2015) Thermoperiodic control of hypocotyl elongation depends on auxin-induced ethylene signaling that controls downstream PHYTOCHROME INTERACTING FACTOR3 activity. Plant Physiol 167(2):517-530.

50. Lin RC, Park HJ, \& Wang HY (2008) Role of Arabidopsis RAP2.4 in regulating lightand ethylene-mediated developmental processes and drought stress tolerance. Mol Plant $1(1): 42-57$.

51. Shin J, Park E, \& Choi G (2007) PIF3 regulates anthocyanin biosynthesis in an HY5dependent manner with both factors directly binding anthocyanin biosynthetic gene promoters in Arabidopsis. Plant J 49(6):981-994.

52. Imaizumi T (2010) Arabidopsis circadian clock and photoperiodism: time to think about location. Curr Opin Plant Biol 13(1):83-89.

53. Nozue K, et al. (2007) Rhythmic growth explained by coincidence between internal and external cues. Nature 448(7151):358-361.

54. Kuno N, et al. (2003) The novel MYB protein EARLY-PHYTOCHROMERESPONSIVE1 is a component of a slave circadian oscillator in Arabidopsis. Plant Cell 15(10):2476-2488. 
1061 55. Rawat R, et al. (2011) REVEILLE8 and PSEUDO-REPONSE REGULATOR5 form a negative feedback loop within the Arabidopsis circadian clock. PLoS Genet 7(3):e1001350.

1064 56. James AB, et al. (2008) The circadian clock in Arabidopsis roots is a simplified slave version of the clock in shoots. Science 322(5909):1832-1835.

57. Silva-Navas J, et al. (2015) D-Root: a system for cultivating plants with the roots in darkness or under different light conditions. Plant J 84(1):244-255.

58. Thimm O, et al. (2004) MAPMAN: a user-driven tool to display genomics data sets onto diagrams of metabolic pathways and other biological processes. Plant J 37(6):914-939.

59. Siddharthan R (2006) Sigma: multiple alignment of weakly-conserved non-coding DNA sequence. BMC Bioinformatics 7:143.

60. Proost S, et al. (2015) PLAZA 3.0: an access point for plant comparative genomics. Nucleic Acids Res 43(Database issue):D974-981.

61. Sturn A, Quackenbush J, \& Trajanoski Z (2002) Genesis: cluster analysis of microarray data. Bioinformatics 18(1):207-208.

62. Tamura K, Stecher G, Peterson D, Filipski A, \& Kumar S (2013) MEGA6: Molecular

63. Finkelstein RR, Gampala SS, \& Rock CD (2002) Abscisic acid signaling in seeds and seedlings. Plant Cell 14 Suppl:S15-45.

64. Weirauch MT, et al. (2014) Determination and inference of eukaryotic transcription factor sequence specificity. Cell 158(6):1431-1443.

66. Kereszt A, et al. (2007) Agrobacterium rhizogenes - mediated transformation of soybean to study root biology. Nature Protocols 2(4):948-952.

65. Taylor-Teeples M, et al. (2015) An Arabidopsis gene regulatory network for secondary cell wall synthesis. Nature 517(7536):571-U307.

67. van der Weele CM, Spollen WG, Sharp RE, \& Baskin TI (2000) Growth of Arabidopsis thaliana seedlings under water deficit studied by control of water potential in nutrientagar media. J Exp Bot 51(350):1555-1562.

68. Verslues PE \& Bray EA (2004) LWR1 and LWR2 are required for osmoregulation and osmotic adjustment in Arabidopsis. Plant Physiol 136(1):2831-2842. 
1092 69. Dinneny JR, et al. (2008) Cell identity mediates the response of Arabidopsis roots to $1093 \quad$ abiotic stress. Science 320(5878):942-945.

1094 70. Armengaud P (2009) EZ-Rhizo software: the gateway to root architecture analysis. Plant 1095 Signal Behav 4(2):139-141.

1096

1097 
bioRxiv preprint doi: https://doi.org/10.1101/2020.03.19.999482; this version posted March 20,2020. The copyright holder for this preprint (which was not certified by peer review) is the author/funder, who has granted bioRxiv a license to display the preprint in perpetuity. It is made available under aCC-BY-NC-ND 4.0 International license.

A
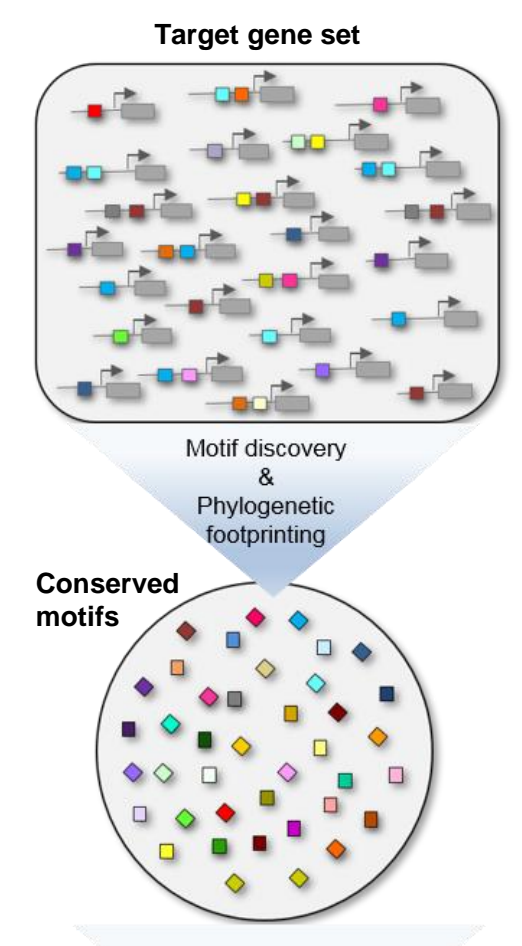

Traceback of motifs in group

\section{Core TFs}

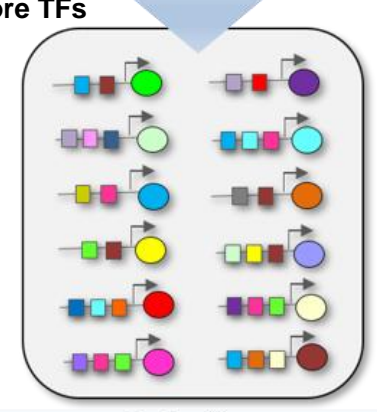

Co-function

\&

TF-DNA interaction analysis

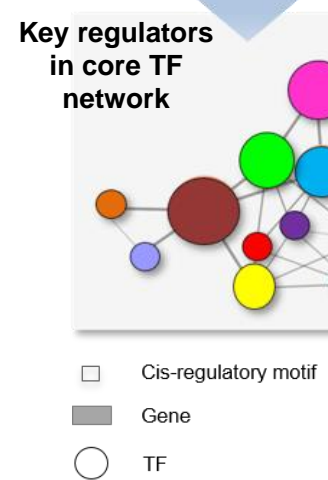

B

Examination of the spatial-temporal gene expression in soybean roots under water deficits using the Affymetrix Soybean GeneChip

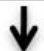

Identification of 279 genes truly regulated by water deficit conditions in soybean primary root

\begin{tabular}{|ccc|}
\hline Co-expression cluster & $\downarrow$ & $\begin{array}{c}\text { MEME de novo motif discovery } \\
\text { length 6-15 bp }\end{array}$ \\
\hline $\begin{array}{c}\text { Identification of de novo } 89 \\
\text { Phylogenetic footprinting } \\
\text { analysis across } 13 \text { species }\end{array}$ & $\downarrow$ & FDR $<0.1$ \\
\hline $\begin{array}{c}\text { Identification of } 49 \text { conserved functional motifs } \\
\text { conserved genes }\end{array}$ & $\downarrow$ & $\begin{array}{c}\text { GO annotations from the } \\
\text { PLAZA 3.0 database }\end{array}$ \\
\hline
\end{tabular}

GO enrichment analysis of conserved motif associated 7954 genes and motifs classification

$\begin{gathered}\text { Focus core TFs based on motifs } \\ \text { combination and the response to both } \\ \text { endogenous and abiotic stimuli response }\end{gathered}$
$\quad \begin{gathered}\text { Root-related transcriptomic data } \\ \text { integration and enrollment of } \\ \text { unannotated TF candidates }\end{gathered}$

Traceback of core transcription factors as signal mediators during water deficits in soybean primary root

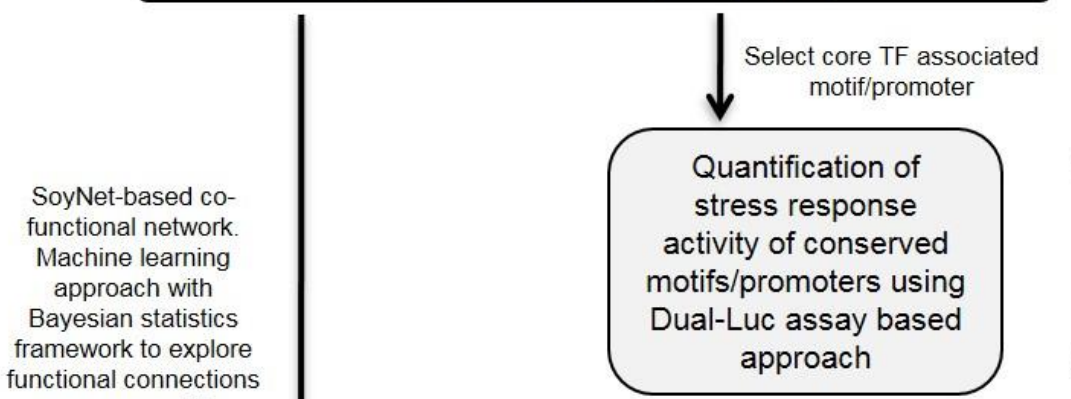

Construction of a root-related water stress responsive TF library including 214 TFs in 22 TF families unctional connections among core TFs

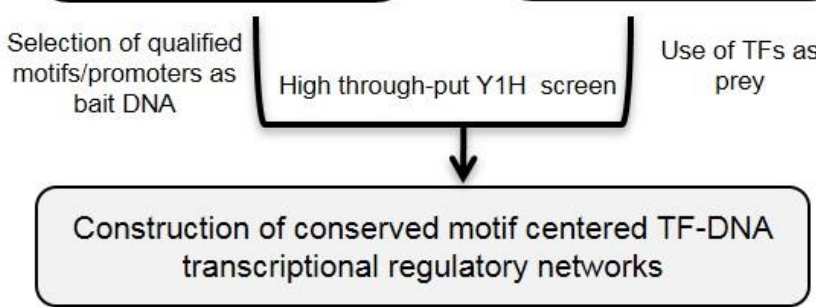

Construction of a TF-TF cofunctional network

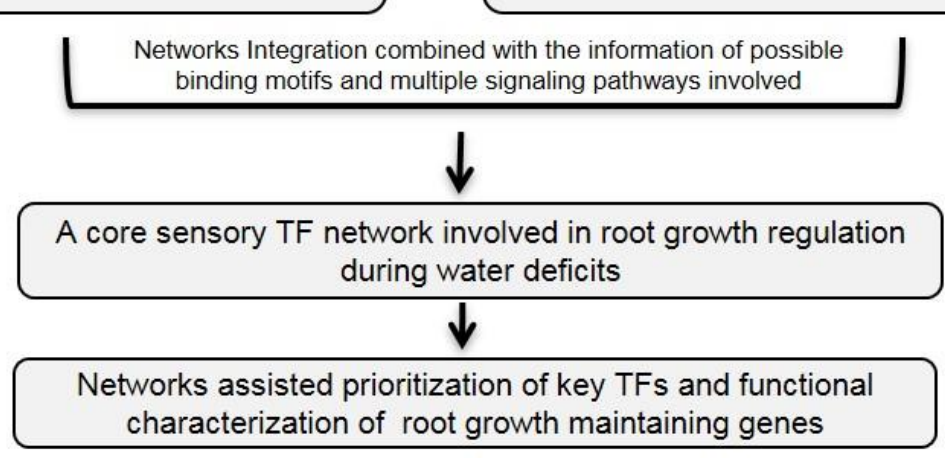

Figure 1. Overview of the workflow used to trace back core TFs and to prioritize key regulators using an assembly strategy. (A) An assembly strategy to trace back core TFs in group and prioritize key regulators. (B) Overview of the workflow used to trace back core TFs and to prioritize key regulators involved in maintaining root growth under water deficit. MEME (Multiple Expectation maximization for Motif Elicitation): a tool for discovering de novo motifs. FDR: false discovery rate. GO: gene ontology. PLAZA: an online platform for plant comparative genomics (http://bioinformatics.psb.ugent.be/plaza/). SoyNet: a database of co-functional networks of G. max genes (www.inetbio.org/soynet). Y1H: yeast one-hybrid. 
bioRxiv preprint doi: https://doi.org/10.1101/2020.03.19.999482; this version posted March 20, 2020. The copyright holder for this preprint (which was pof certified by peer review) is the author/funder, who has granted bioRxiv a license to display the preprint in perpetuity. It is made avBiA\&laBîlder aCC-BY-NC-ND 4.0 International license.

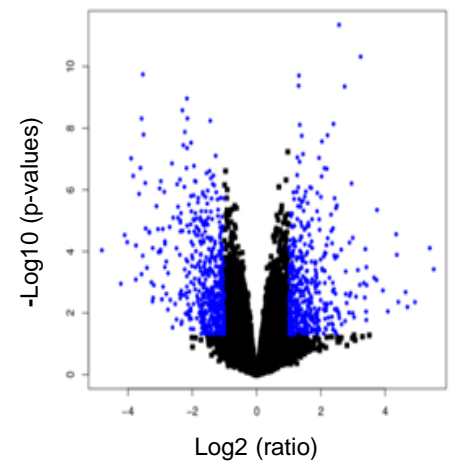

D5hR2

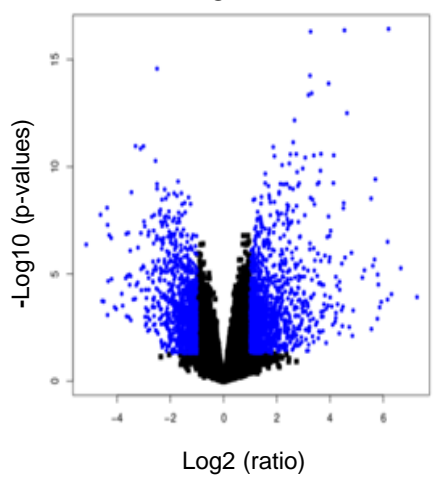

C
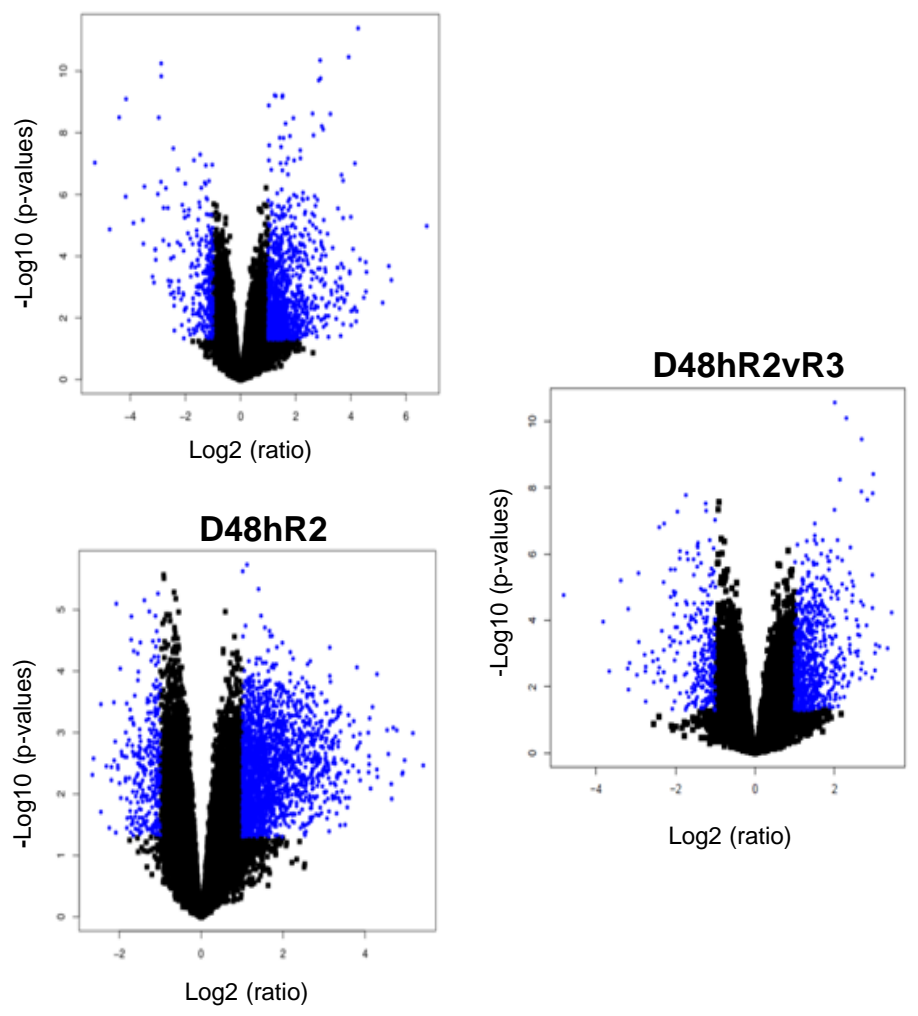

B

D
D48hR2 overlapped with D48hR2vR3

\# genes $=279$

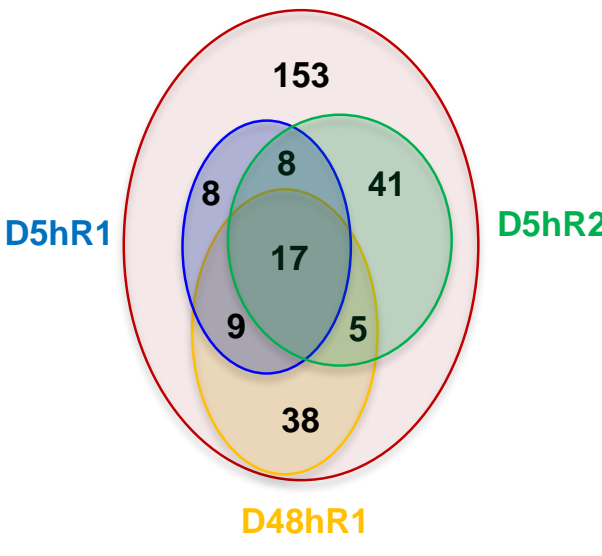

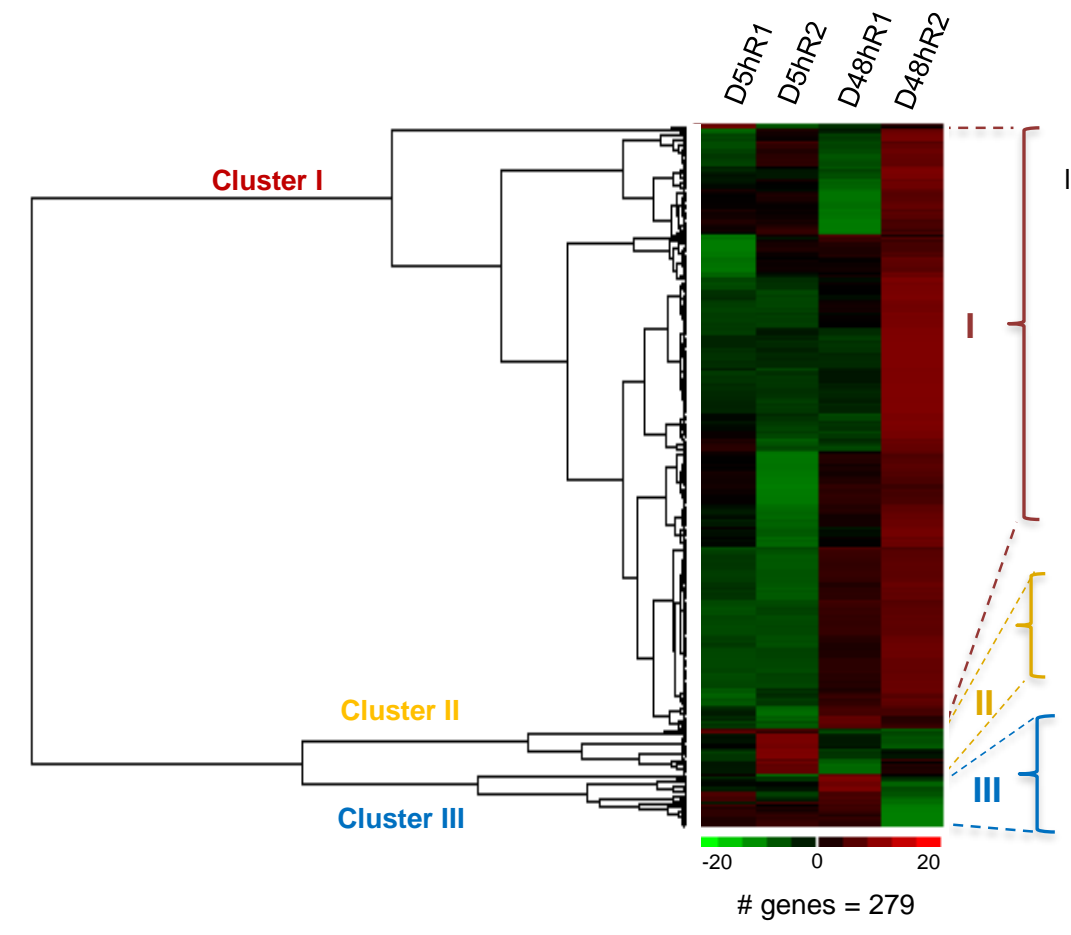

MapMan BIN enrichment

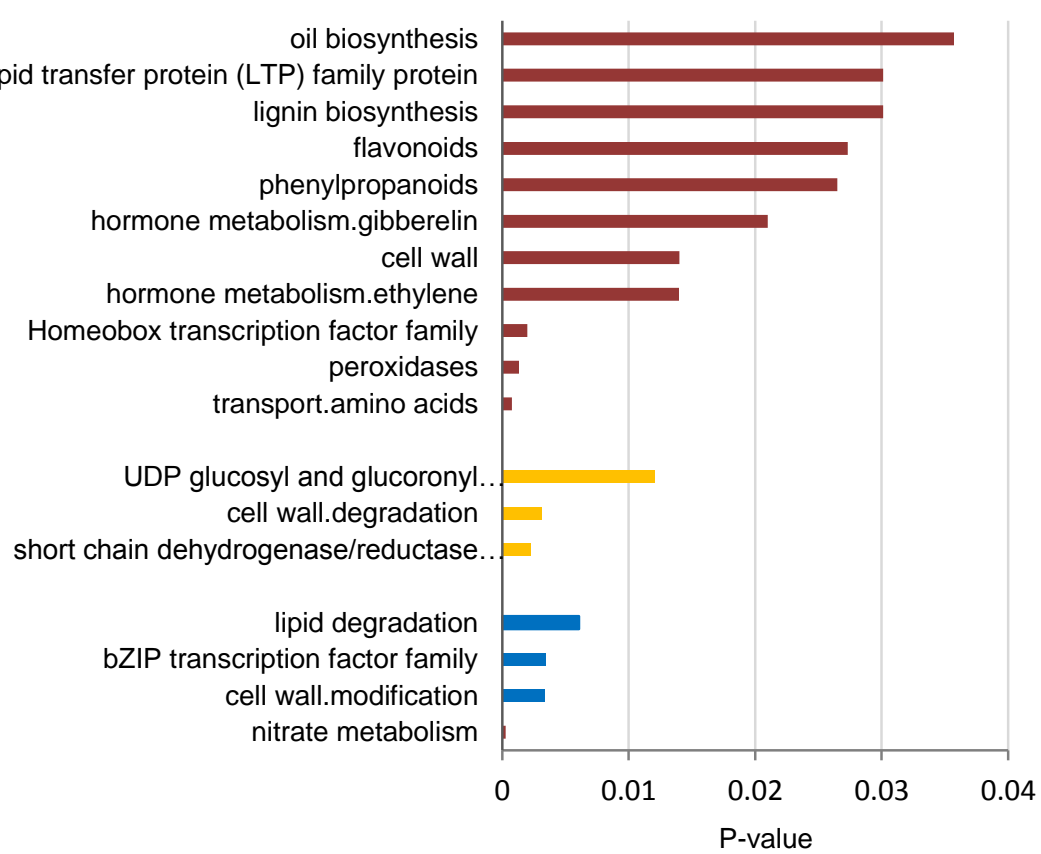

Figure 2. Identification of $\mathbf{2 7 9}$ genes spatially-temporally regulated by water deficit conditions in soybean primary roots.

(A) Volcano plots showing significant changes in gene expression in soybean root regions R1, R2 and R3 in response to $5 \mathrm{~h}$ and 48 $\mathrm{h}$ of water stress treatment. The transcripts were divided into five groups according to their expression profiles across the comparisons: D5hR1, D5hR2, D48hR1, D48hR2 and D48hR2vR3. The Benjamini-Hochberg p-values were plotted against the fold change in gene expression for all genes. Log2 fold change ( $x$-axis) versus negative log10 $p$-values ( $y$-axis). Blue indicates transcripts with fold-changes of $\geq|2.0|$ and $p$-values of $\leq 0.05$ with respect to the corresponding well-watered control.

(B) Venn diagram illustrating the number of stress-responsive genes (279 in total) with significantly altered ( $\geq|2.0|$ fold, the Benjamini-Hochberg p-values $\leq 0.05$ ) expression in both D48hR2 and 48hR2vR3 (red) and the number of genes shared with D5hR1 (blue), D5hR2 (green) and D48hR1 (yellow).

(C) Hierarchical tree clustering analysis of 279 differentially expressed genes in different soybean root regions after the primary root was deprived of water for $5 \mathrm{~h}$ and $48 \mathrm{~h}$. Hierarchical clustering of the 279 selected genes yielded three gene clusters: I, II and III.

(D) Overrepresented MapMan bins assigned to the genes in each of three clusters. Enrichment of each category was tested with Fisher's exact test ( $p$-values $\leq 0.05$ ) 


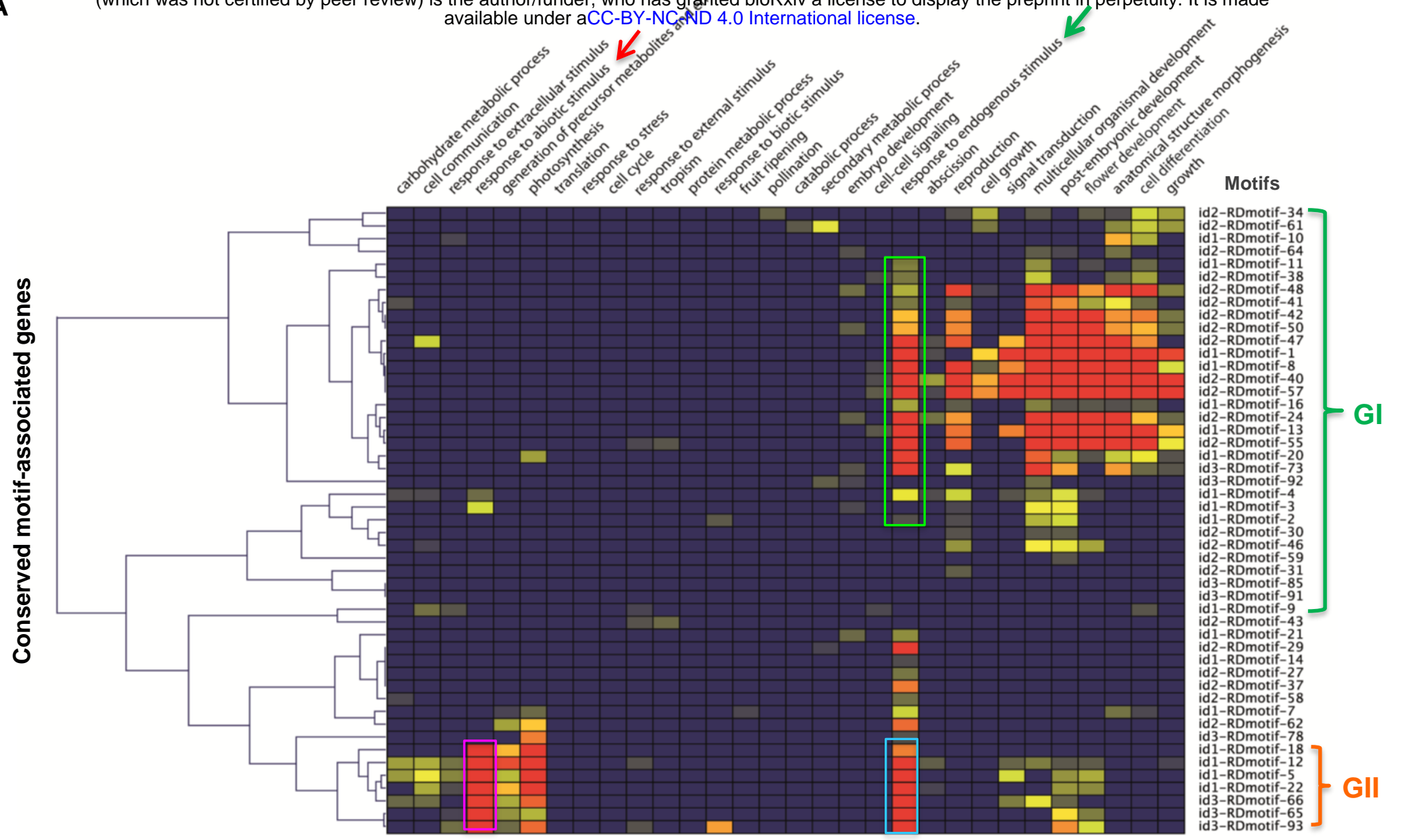

B

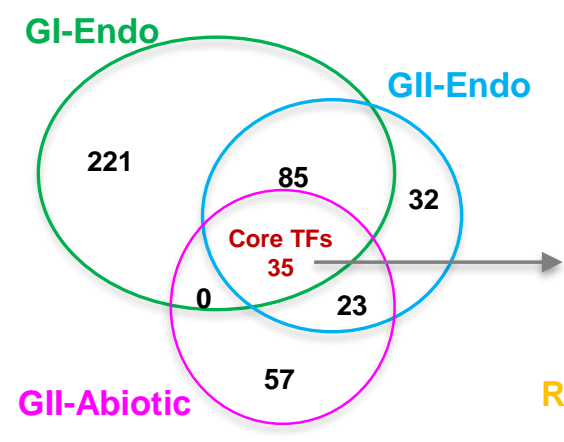

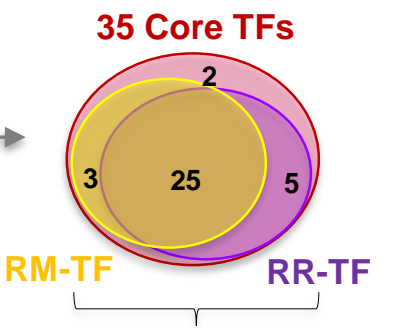

33 rdc TFs

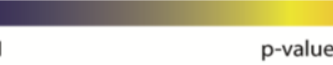

p-value
\# genes $=7954$

\section{Enrichment of combination with conserved motif RDmotif-4 :GGG.}

\begin{tabular}{|c|c|c|c|c|}
\hline \multirow{3}{*}{$\begin{array}{l}\text { Rdmotif-22 } \\
C C A C G=G\end{array}$} & \multicolumn{2}{|c|}{ Group I } & \multicolumn{2}{|c|}{ Group II } \\
\hline & Fold & $p$-value & Fold & $p$-value \\
\hline & 2.09 & $4.88 \mathrm{E}-02$ & 5.18 & $1.86 \mathrm{E}-05$ \\
\hline $\begin{array}{l}\text { Rdmotif-8 } \\
\text { AGAGAGAGAG }\end{array}$ & 3.56 & $5.28 \mathrm{E}-05$ & 4.28 & 2.25E-04 \\
\hline $\begin{array}{l}\text { Rdmotif-24 } \\
C C=C=C\end{array}$ & 2.62 & 3.57E-04 & 3.98 & $1.03 \mathrm{E}-05$ \\
\hline 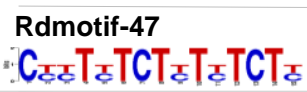 & 4.39 & $4.39 \mathrm{E}-03$ & 7.64 & 3.72E-04 \\
\hline
\end{tabular}

Figure 3. Identification of root-related core TFs that function in the response to endogenous and abiotic stimuli under water-deficit conditions.

(A) GO enrichment analysis of the 7954 conserved target genes associated with the 49 conserved motifs genome-wide. The vertical axis shows the conserved motifs and the horizontal axis shows the enriched GO terms for the target genes. Conserved motifs and associated target genes were classified into two groups based on GO annotations. Group I (Gl): Genes and motifs involved in plant growth and development. Group II (GII): Genes and motifs involved in responses to abiotic stress. Green arrow: response to endogenous stimulus. Red arrow: response to abiotic stimulus. Green box contains genes annotated with the GO term "response to endogenous stimulus" in GI. Blue box contains genes annotated with the GO term "response to endogenous stimulus" in GIl. Pink box contains genes annotated with the GO term "response to abiotic stimulus" in GIl. GO: gene ontology. (B) Venn diagram showing the 35 core TFs that overlap in all three groups of TFs. GI-Endo (green): TFs associated with GO term "response to endogenous stimulus" in Group I. Gll-Endo (blue): TFs associated with GO term "response to endogenous stimulus" in Group II. GII-Abiotic (pink): TFs associated with GO term "response to abiotic stimulus". The expression of 33 rdc TFs among the 35 core TFs is either significantly altered in the root elongation region-specific microarray gene expression profile (RM-TF, yellow) or in the RNA-seq transcriptome profiling data for soybean primary roots under various water deficit conditions (RR-TF, purple).

(C) Enrichment of the motifs combined with conserved motif RDmotif-4 in the promoters of genes in Group I or Group II with the GO term "response to endogenous stimulus". Group I represents the genes shown in the green box in (A). Group II represents the genes shown in the blue box in $(A)$. 
bioRxiv preprint doi: $\mathrm{https}$ //doi.org/10.1101/2020 03.19 .999482 ; this version posted March 20,2020 . The copyright holder for this preprint (which was not certified by peer review) is the author/funder, who has granted bioRxiv a license to display the preprint in perpetuity. It is made available under aCC-BY-NC-ND 4.0 International license.

A

A 96-well plate arrayed TF library including 214 root drought-related TFs for Y1H screen

\begin{tabular}{lcc}
\hline TF family & Number & Percentage \\
MYB & 31 & $14 \%$ \\
bHLH & 26 & $12 \%$ \\
AP2 & 25 & $12 \%$ \\
HB & 14 & $7 \%$ \\
NAC & 13 & $6 \%$ \\
bZIP & 10 & $5 \%$ \\
NF-YB & 9 & $4 \%$ \\
Others & 86 & $40 \%$ \\
\hline
\end{tabular}

B

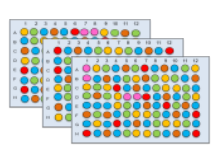

TF library arrayed in 96-well plates

Prey protein

\section{Bait DNA}

Motif repeats

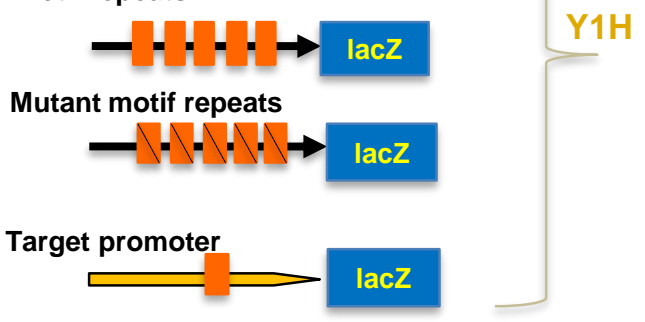

Y1H screening against 214 TF array

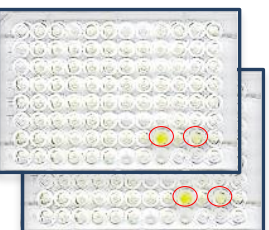

$1^{\text {st }}$ screen

$\downarrow$

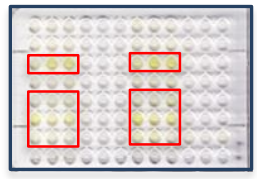

$2^{\text {nd }}$ screen

C

D
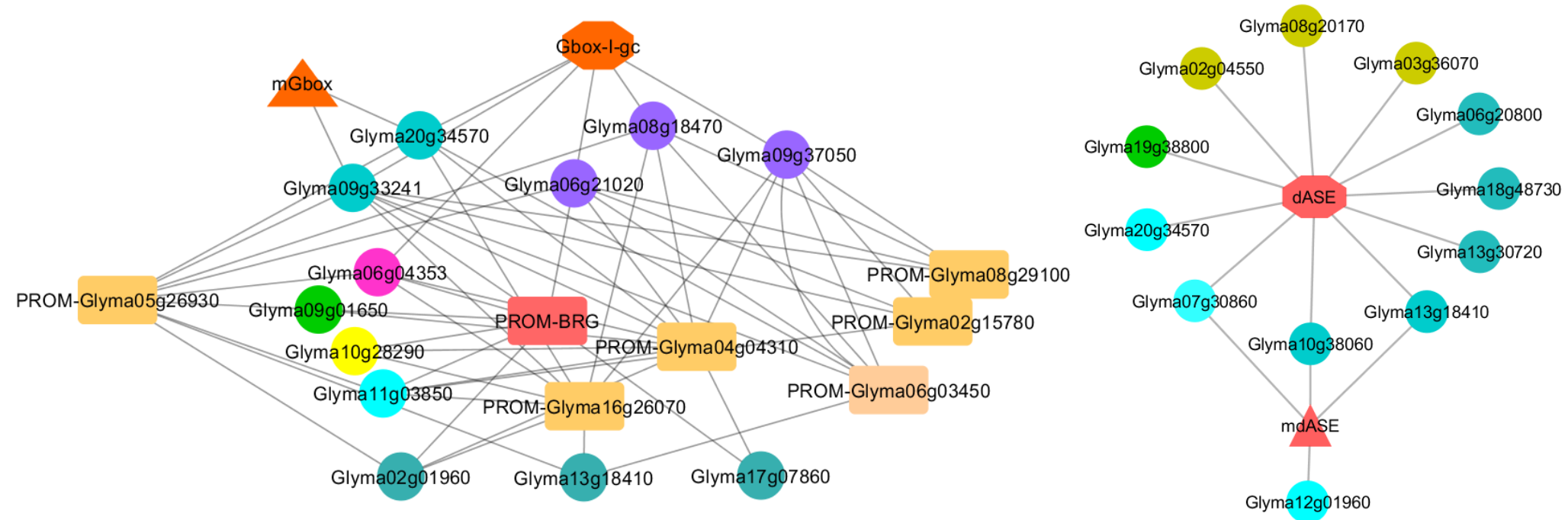

Figure 5. Construction of conserved motif-centered TF-DNA interaction networks.

(A) A drought-related transcription factor library consisting of 214 TFs in 22 TF families, with a color-coded legend.

(B) Schematic diagram of the conserved motif-centered $\mathrm{Y} 1 \mathrm{H}$ assays. Bait DNAs included conserved motif repeats of interest, mutant conserved motif repeats and associated promoter fragments. Each TF was tested in quadruplicate. Red boxes show positive interactions.

(C) Conserved RDmotif22-centered TF-DNA interaction network. Gbox-I-gc represents RDmotif22. mGbox represents the mutated G-box. PROM-BRG represents the RDmotif22-associated promoter fragment of $B R G$. PROM-

Glyma06g03450 are negative binding promoters. In the network, six promoter fragments were classified into different groups based on their preferred binding TFs.

(D) Conserved dASE motif-centered TF-DNA interaction network. mdASE represents the mutated dASE motif.

(C)-(D) Octagon: conserved motif. Triangle: mutated conserved motif. Rectangle: promoter fragments. Circle: interacting TF. The color of each oval represents the same TF family as in (A). 
Betweenness Centrality

\begin{tabular}{l|}
\hline Min=0.00 Max=0.45 \\
\hline W: Water deprivation response \\
D: Response to desiccation \\
S: Salt stress response \\
O: Osmotic stress response \\
C: Cold stress response \\
L: Response to light signal \\
I: Circadian Rhythm related \\
F: Positive regulation of flavonoid \\
biosynthetic process \\
P: Response to superoxide \\
G: Gravitropism related \\
R: lateral root development
\end{tabular}

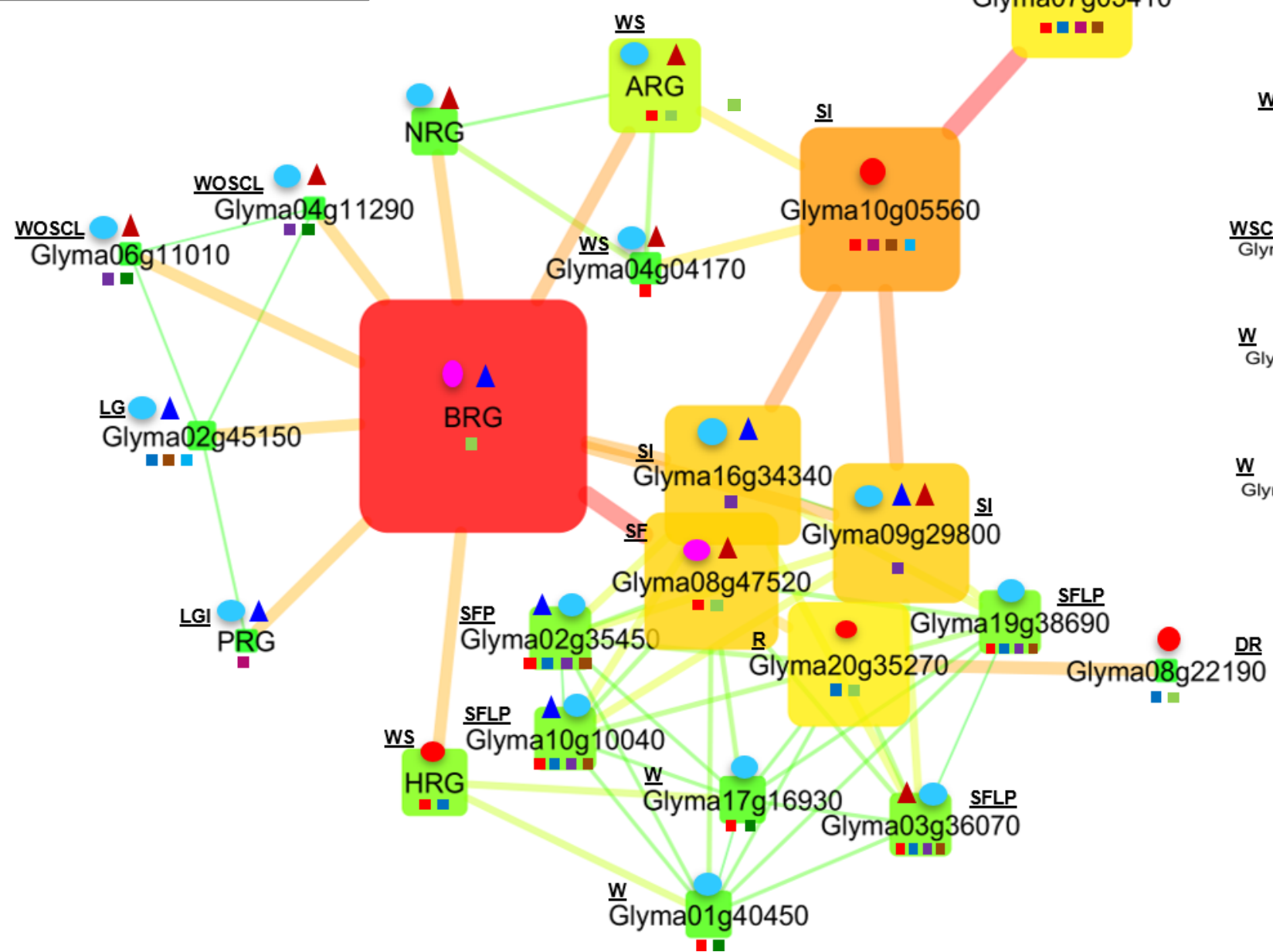

$\triangle$ RD22motif site associated with binding protein ARG

$\Delta$ dASE motif site associated with binding protein Glyma03g36070

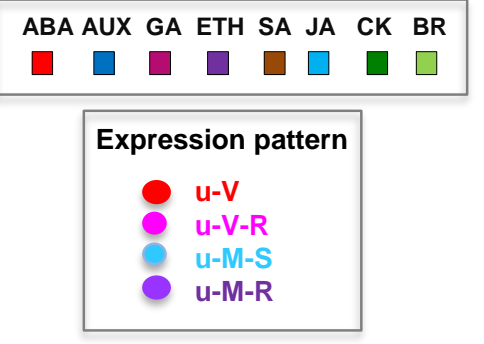

ABA AUX GA ETH SA JA CK BR

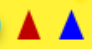

Glyma07g05410

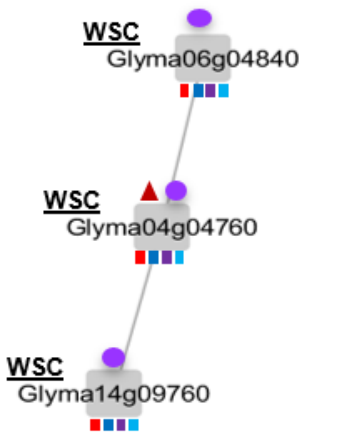

R

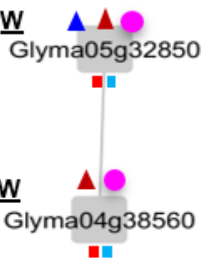

Figure 6. Core sensory TF network that functions in soybean primary roots under water-deficit conditions. Gene IDs are shown in boxes. The node size corresponds to the strength of the combined evidence from SoyNet and the $\mathrm{Y} 1 \mathrm{H}$ assay. The color intensity indicates the betweenness of centrality in the network. Edge betweenness is indicated by line thickness. Different abiotic stress responses and the other pathways involved are represented by underlined capital letters associated with each core TF. Small squares of different colors connected with each Gene ID box represent different hormone responses. ABA, abscisic acid. AUX, auxin. GA, gibberellic acid. ETH, ethylene. SA, salicylic acid. JA, jasmonic acid. CK, cytokinin. BR, brassinosteroid. Triangles represent the motifs on the promoters of core TFs showing possible binding sites, including motifs RDmotif22 and dASE site. Ovals attached to each Gene ID represent the gene expression pattern under different water-deficit levels in soybean primary roots. A heatmap of gene expression levels is shown in Figure S3A. 
bioRxiv preprint doi: https://doi.org/10.1101/2020.03.19.99982; this version posted March 20, 2020. The copyright holder for this preprint (which was not certified by peer review) is the author/funder, Who has granted bioRxiv a license to display the preprint in perpetuity. It is made available under aCC-BY-NC-ND 4.0 International license.
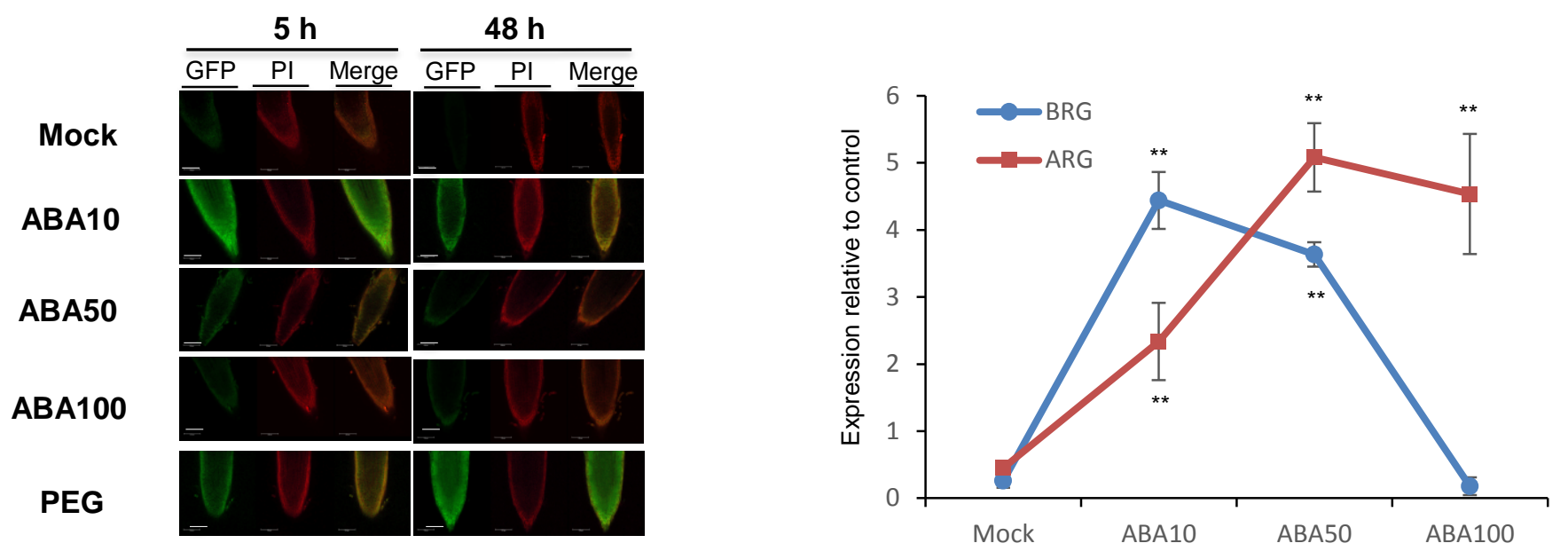

C

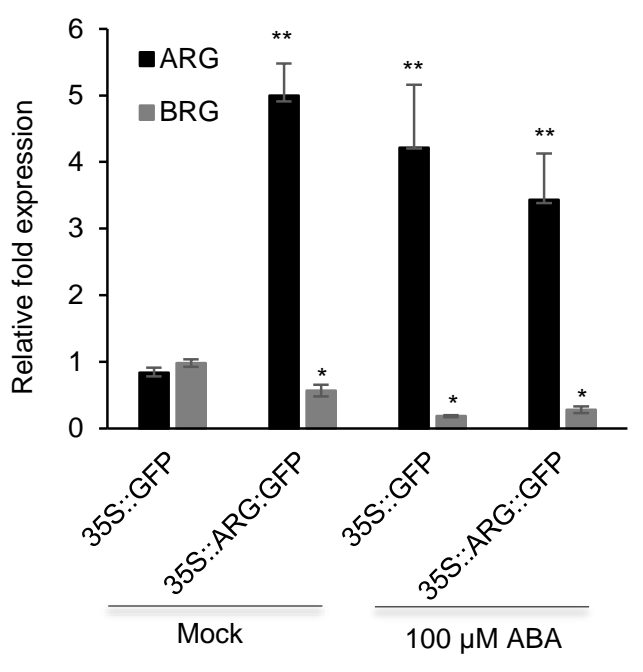

D

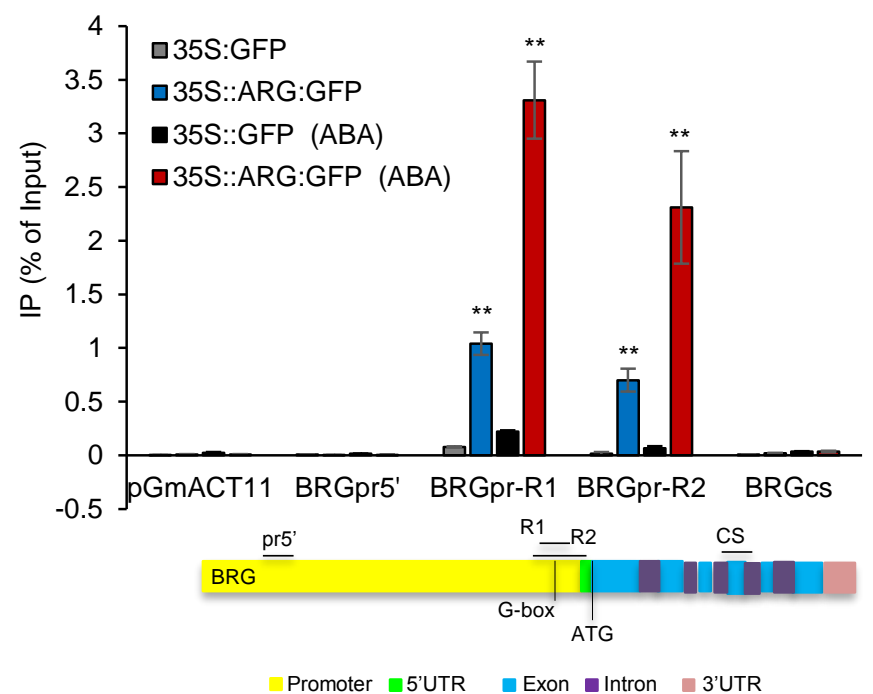

Figure 7. ARG directly binds to the $B R G$ promoter and represses its expression

(A) promBRG::GFP expression after ABA or PEG treatment. Soybean transgenic hairy root tips (PROM-BRG::GFP) used for scanning were incubated on $1 / 2 \mathrm{MS}$ solid medium (3\% sucrose, $0.6 \%$ agar) containing $10 \mu \mathrm{M}, 50 \mu \mathrm{M}$ and 100 $\mu \mathrm{M}$ ABA or PEG-infused medium (-1.7 MPa) at $5 \mathrm{~h}$ and $48 \mathrm{~h}$. (representative images are shown; $\mathrm{n}=4$ per line, with three independent lines). Cell walls were stained with propidium iodide (PI, red). Scale bar: $100 \mu \mathrm{m}$.

(B) The expression of $B R G$ and $A R G$ is inversely correlated with $A B A$ levels. $A R G$ and $B R G$ expression levels in soybean roots relative to the Glyma18g52780 (GmACT11) control were evaluated after 7 days on $1 / 2$ MS solid medium supplemented with $10 \mu \mathrm{M}, 50 \mu \mathrm{M}$ and $100 \mu \mathrm{M}$ ABA. ( $\mathrm{n}=3$ biological replicates with 3 technical replicates; mean \pm SE). Significance relative to mock-treated root samples was determined by two-tailed Welch's $t$-test : double asterisks denote significance level at $\mathrm{P}<0.001$.

(C) $A R G$ and $B R G$ expression relative to the GmACT11 control in transgenic 35S::ARG::GFP soybean roots relative to the empty vector control (35S::GFP). Relative $A R G$ and $B R G$ expression levels were evaluated after 3 days with or without $100 \mu \mathrm{M}$ ABA treatment ( $\mathrm{n}=3$ biological replicates with 3 technical replicates. mean $\pm S E$ ). Significance relative to the empty vector control in mock samples was determined by two-tailed Welch's $t$-test: single and double asterisks denote significance level at $P<0.01$ and $P<0.001$, respectively.

(D) ChIP-qRT-PCR of four areas of the BRG genomic regions. Values for 35S::ARG::GFP and 35S::GFP were calculated as the percentage of the input after 3 days with or without $100 \mu \mathrm{M} A B A$. The relative placement of the amplified regions is marked by black lines. (Regions: BRGpr5' = -1967/-1882, BRGpr-R1 = -124/-65, BRGpr-R2 = $120 /-37$, BRGcs $=+1004 /+1079$, base pairs from the transcriptional start site). $n=3$ biological replicates with 3 technical replicates (mean $\pm \mathrm{SE}$ ). Significance relative to the control (promoter region of GmACT11; pGmACT11) was determined by two-tailed Welch's $t$-test : double asterisks denote significance level at $P<0.001$. 
bioRxiv preprint doi: https://doi org/10.1101/2020.03 19 999482; this version posted March 20, 2020. The copyright holder for this preprint (which was not certified by peer review) is the thor/funder, who has granted bioRxiv a license to display the preprint in perpetuity. It is made avaifâble under aCC-BY-NC-ND 4.0 International license.C

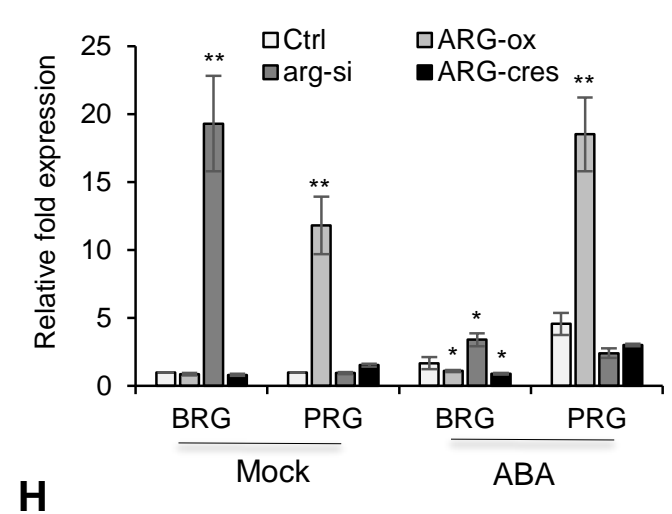

H

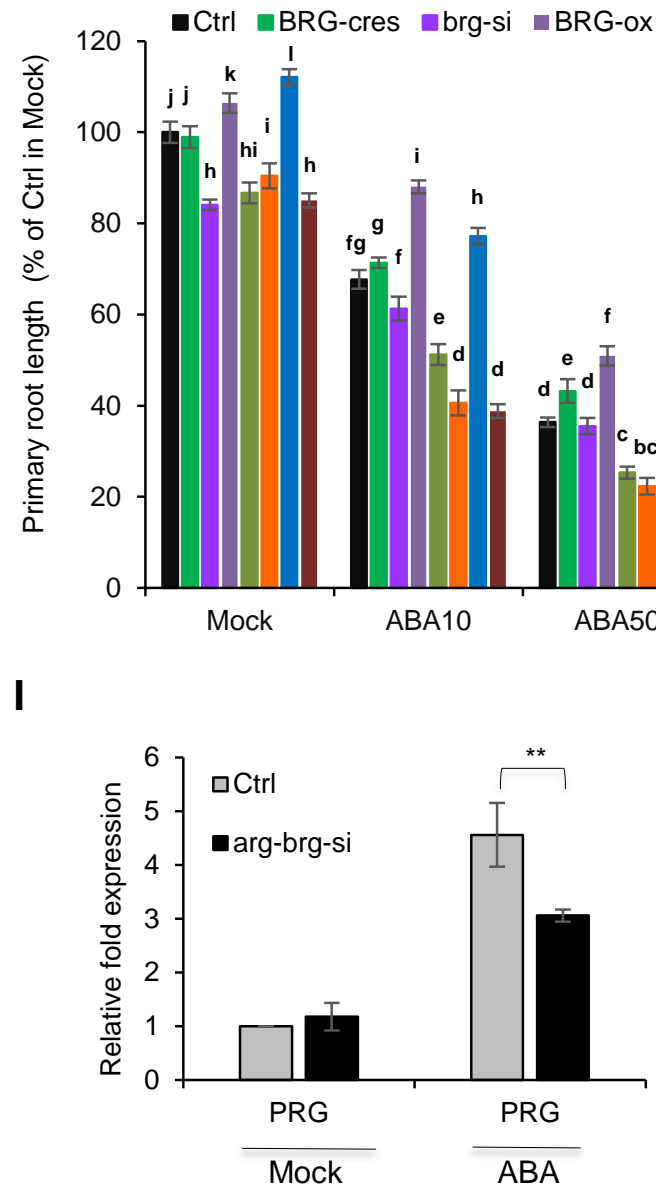

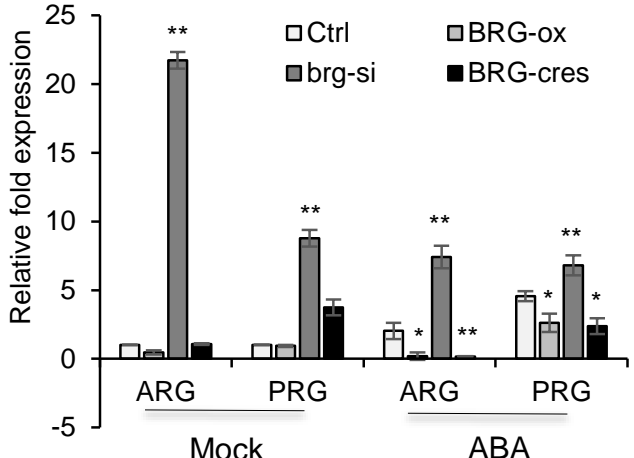

ARG-cres arg-si $\quad$ ARG-ox arg-brg-si

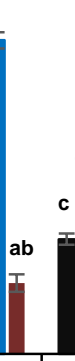

ABA100

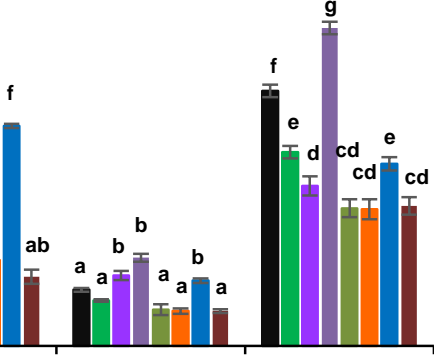

PCZ-BL

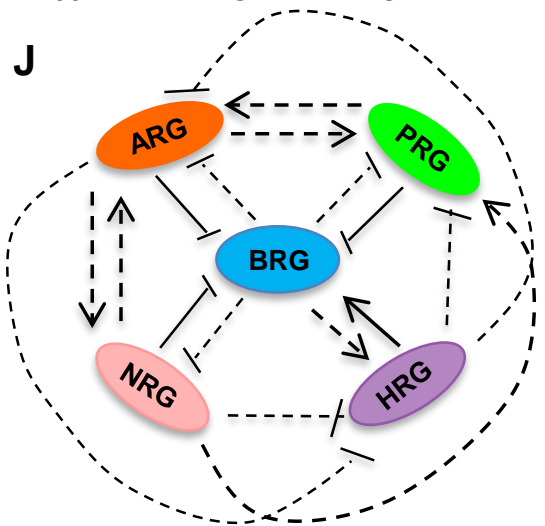

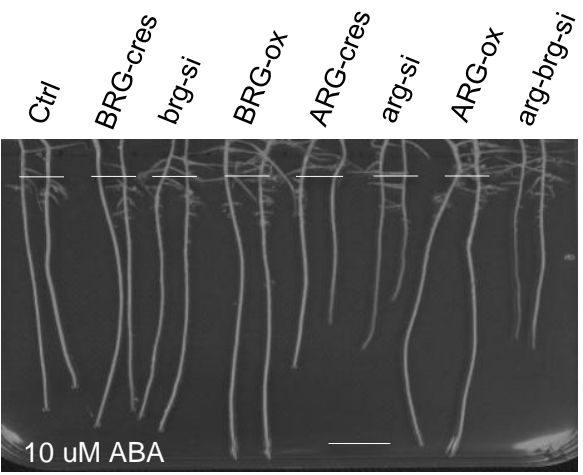

D

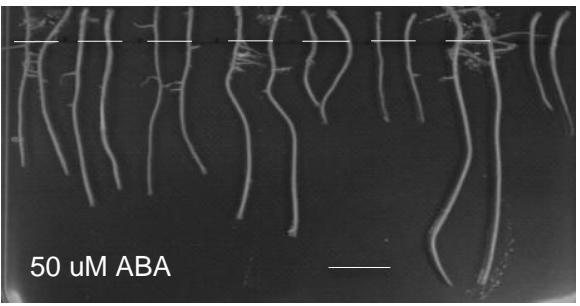

E

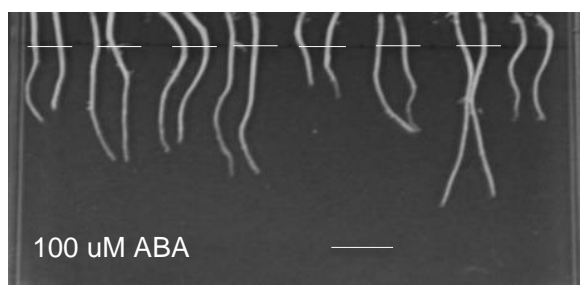

$\mathbf{F}$

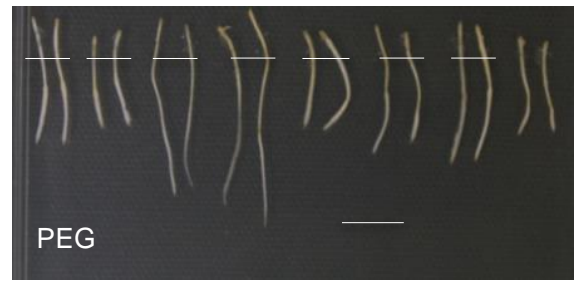

G

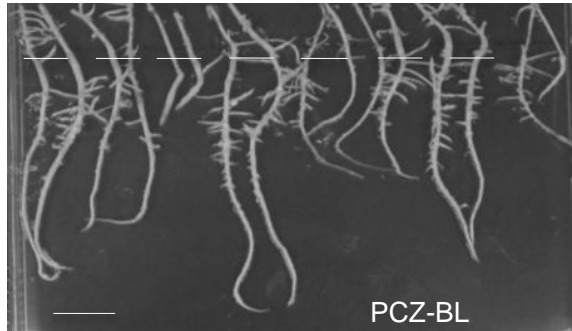

Figure 8. ARG and BRG antagonistically regulate $P R G$, and their effect on root growth maintenance.

(A) RT-qPCR showing $B R G$ and $P R G$ transcript levels in root samples of the empty vector control (Ctrl), ARG-ox, arg-si and ARG-cres mutants treated for 3 days with $100 \mu \mathrm{M} \mathrm{ABA}$ relative to mock-treated roots.

(B) RT-qPCR showing ARG and PRG transcript levels in root samples from Ctrl, ARG-ox, arg-si and ARG-cres plants treated for 3 days with $100 \mu \mathrm{M}$ ABA relative to mock-treated roots.

(A)-(B) Expression was normalized to the GmACT11 reference gene ( $n=3$ biological replicates with 3 technical replicates; mean $\pm S E)$. Significance relative to the empty vector control was determined by two-tailed Welch's $t$-test: single and double asterisks denote significance level at $\mathrm{P}<0.05$ and $P<0.001$, respectively.

(C)-(G) Differential growth responses in primary roots of BRG-ox, brg-si, BRG-cres, ARG-ox, arg-si, ARG-cres and arg-brg-si after 7 days of treatment with $10 \mu \mathrm{M}$ ABA, $50 \mu \mathrm{M}$ ABA, $100 \mu \mathrm{M}$ ABA and PEG (-1.7 Mpa). PCZ-BL treatment involved 4 days of $5 \mathrm{nM}$ BL treatment after 3 days of $10 \mu \mathrm{M}$ PCZ treatment. Scale bar: $1 \mathrm{~cm}$.

(H) Quantification of the primary root growth of BRG-ox, brg-si, BRG-cres, ARG-ox, arg-si, ARG-cres and arg-brg-si in response to $10 \mu \mathrm{M}, 50 \mu \mathrm{M}$,

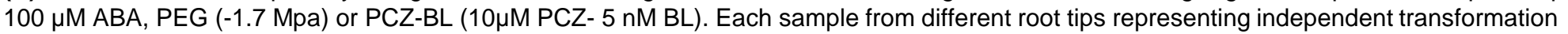
events (mean $\pm S E ; n=15$ ). Comparisons are made between empty vector control plants and mutants prepared using the same growth conditions and the same treatments. Bars with the same letter are not significantly different (two-way ANOVA + Tukey HSD, P < 0.05, ANOVA table in Table S12 showing a significant genotype : hormone/PEG treatment interaction term) in the response to PCZ-BL, ABA and PEG treatment.

(I) RT-qPCR showing PRG transcript levels in root samples of the control (Ctrl) and the arg-brg-si mutant treated for 3 days with $100 \mu \mathrm{M}$ ABA relative to mock-treated roots. Expression was normalized to the GmACT11 reference gene ( $n=3$ biological replicates with 3 technical replicates; mean \pm $\mathrm{SE})$. Significant differences relative to the empty vector control were determined using two-tailed Welch's $t$-test ; double asterisks denote a significance level at $P<0.001$.

(J) Model depicting the possible feedback and feed-forward loops among ARG, BRG, PRG, NRG and HRG. Black lines indicate transcriptional activation (arrows) or inhibition (bar ends). Solid lines represent direct interaction. Dotted lines represent unresolved or indirect relationships. 
bioRxiv preprint doi: https://doi.org/10.1101/2020.03.19.999482; this version posted March 20, 2020. The copyright holder for this preprint (which was not certified by peer review) is the author/funder, who has granted bioRxiv a license to display the preprint in perpetuity. It is made

A

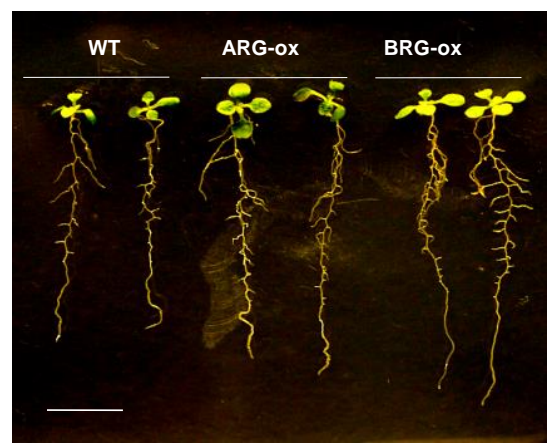

C

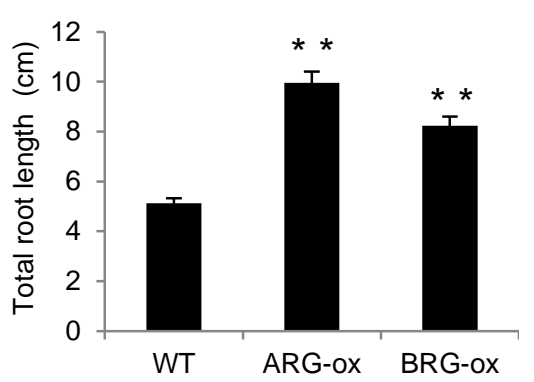

B

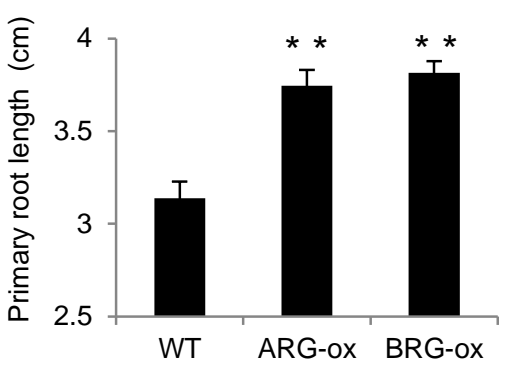

D

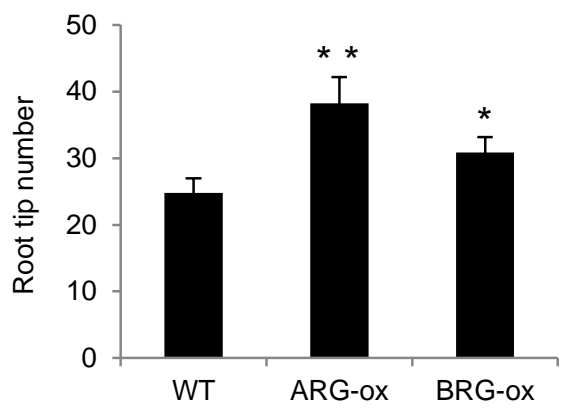

E

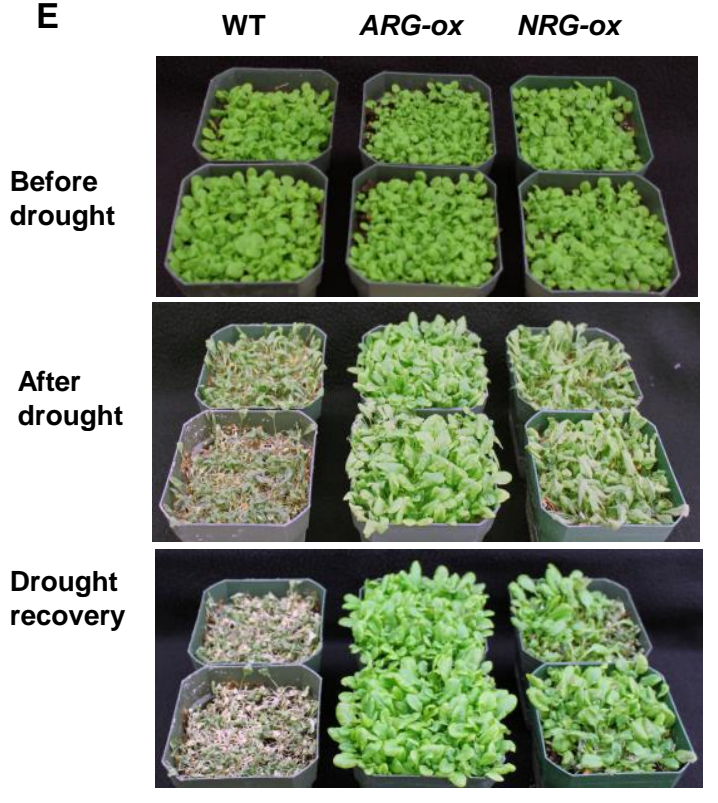

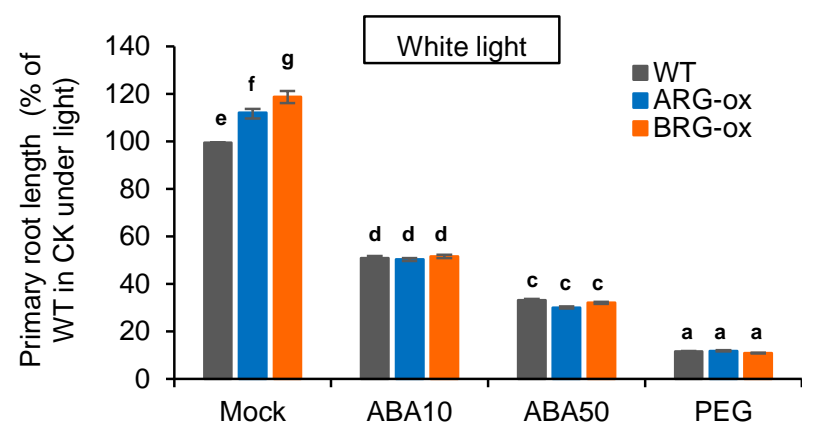
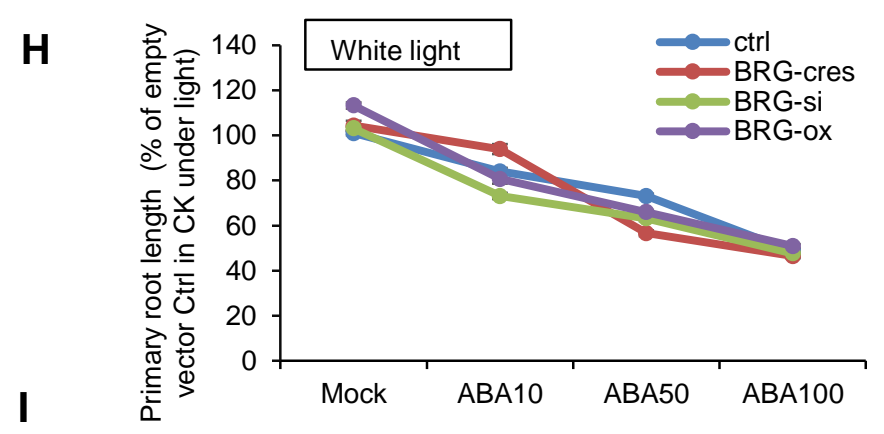

G
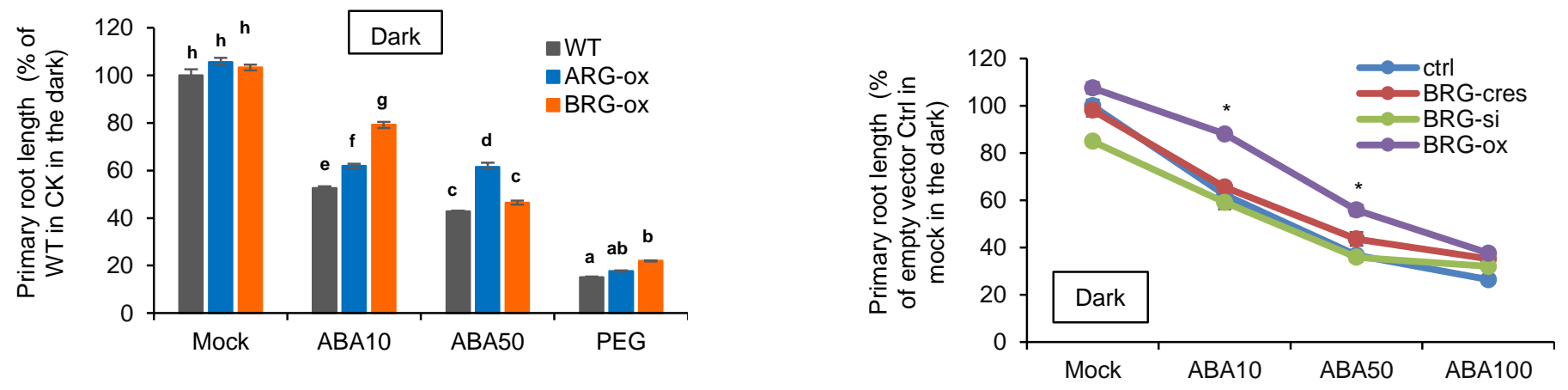

Figure 9. BRG and ARG have an ABA dose-dependent effect on dark-grown root growth.

(A) Increased primary root length and root tip number of $B R G$-ox and $A R G$-ox Arabidopsis plants. Scale bar: $1 \mathrm{~cm}$.

(B)-(D) Primary root length, total root length and number of root tips in BRG-ox and ARG-ox plants compared with WT (Col-0). The germinated seedlings were transferred to medium in square Petri dishes and grown vertically in long days for 7 days. Primary root length, total root length and root tip number were measured (mean $\pm S E ; n=18-24)$. Significance was determined by two-tailed Welch's $t$-test: single and double asterisks denote significance level at $\mathrm{P}<0.01$ and $\mathrm{P}<0.001$, respectively.

(E) Transgenic Arabidopsis ARG-ox and NRG-ox plants are drought resistant. Fourteen-day-old T3 transgenic Arabidopsis plants were well-watered for 12 days, after which water was withheld for 12 days, followed by re-watering for 5 days.

(F)-(G) Quantification of Arabidopsis WT, $A R G$-ox and BRG-ox primary root length in response to $10 \mu \mathrm{M}$ ABA, $50 \mu \mathrm{M}$ ABA or PEG $(-1.7 \mathrm{Mpa})$ under continuous light and in the dark. The comparisons are between WT plants and mutant plants under the same growth conditions and the same treatment (mean $\pm S E ; n=18$ ). Bars with the same letter are not significantly different (two-way ANOVA + Tukey HSD, P < 0.01, ANOVA table in Table S13 showing a significant genotype : ABA/PEG treatment interaction term) in the response to $A B A$ and $P E G$ treatment.

(H)-(I) Response of BRG-cres, brg-si and BRG-ox transgenic soybean roots to $10 \mu \mathrm{M}$ ABA, $50 \mu \mathrm{M}$ ABA and $100 \mu \mathrm{M}$ ABA after 7 days of culture under continuous light or in the dark. Two independent experiments were performed, each sample from different primary roots from independent transformation events (mean $\pm S E ; n=12$ ). Significance was determined by two-tailed Welch's $t$ test: single asterisks denote significance level at $P<0.01$. 


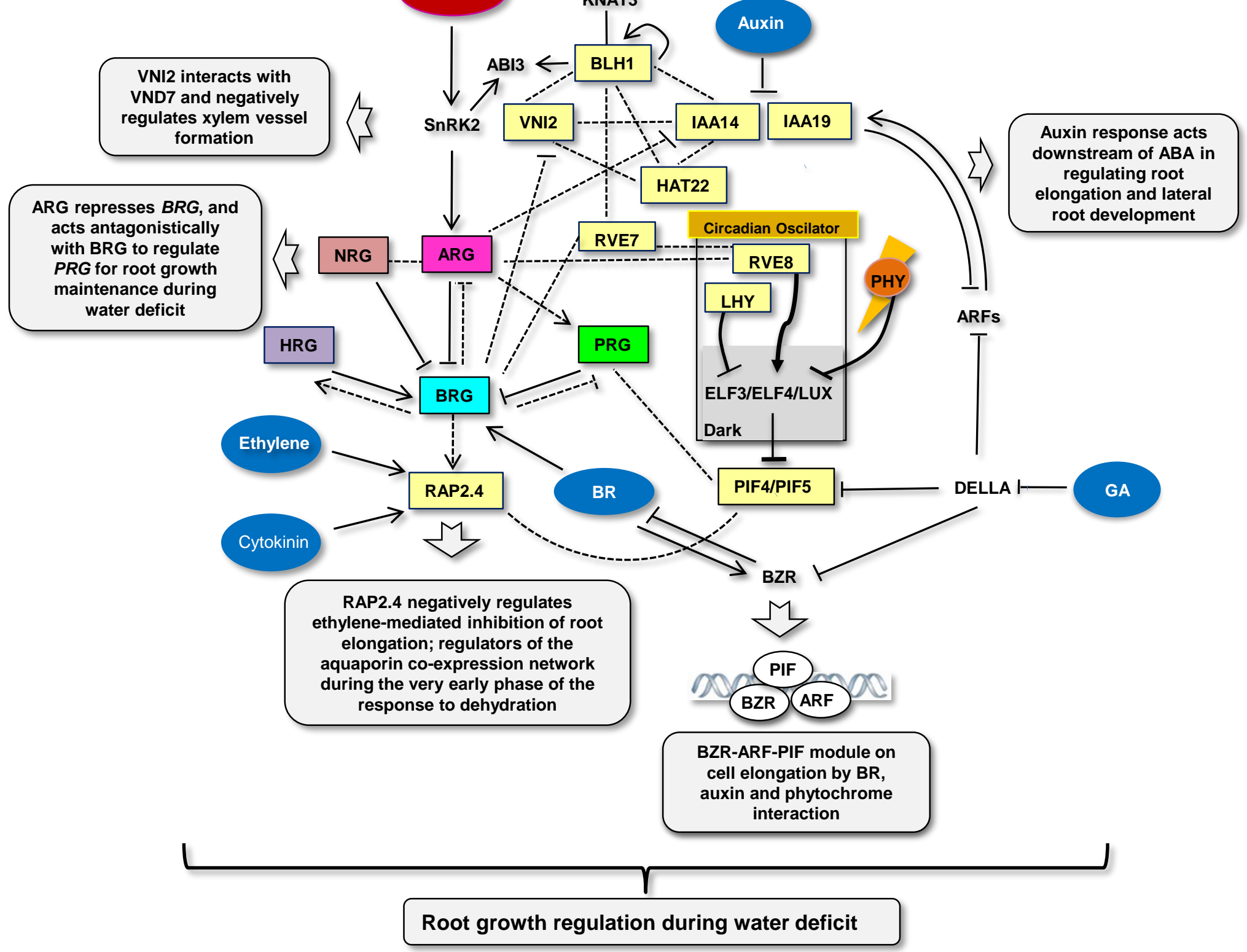

Figure 10. Core sensory TF network integrating hormonal and environmental signaling in roots under water deficit conditions. Core TFs mutually regulate each other, acting as convergence points in the crosstalk among ABA, auxin, GA, $\mathrm{BR}$, ethylene, cytokinin and light signaling to regulate root growth in response to varying water deficit conditions. The genes in boxes are derived from our core sensory network. The genes in yellow boxes and listed outside the boxes are putative orthologs of Arabidopsis genes that are thought to function in the multi-hormonal interaction involved in root growth regulation or the cell elongation module. Hormones are shown in ovals. Black lines indicate known interactions based on published data and direct interactions confirmed by experimental evidence in this study. Black dotted lines represent unresolved relations or indirect interactions awaiting additional investigation. Lines represent transcriptional activation (arrows) or inhibition (bar ends). Functional descriptions are indicated in grey boxes. 